A CHARACTERIZATION OF A DUAL CHAMBERED, TWO PHASE SEPARATOR

\author{
A Thesis \\ by \\ CASEY KLEIN \\ Submitted to the Office of Graduate Studies of \\ Texas A\&M University \\ in partial fulfillment of the requirements for the degree of \\ MASTER OF SCIENCE
}

December 2009

Major Subject: Nuclear Engineering 


\title{
A CHARACTERIZATION OF A DUAL CHAMBERED, TWO PHASE SEPARATOR
}

\author{
A Thesis \\ by \\ CASEY KLEIN \\ Submitted to the Office of Graduate Studies of \\ Texas A\&M University \\ in partial fulfillment of the requirements for the degree of \\ MASTER OF SCIENCE
}

\begin{abstract}
Approved by:
Chair of Committee, Frederick Best

Committee Members, Gerald Morrison

Karen Vierow

Head of Department, Raymond Juzaitis
\end{abstract}

December 2009

Major Subject: Nuclear Engineering 


\begin{abstract}
A Characterization of a Dual Chambered, Two Phase Separator. (December 2009)

Casey Klein, B.S., Texas A\&M University

Chair of Advisory Committee: Dr. Frederick Best
\end{abstract}

A new two phase separator for use in space applications has been invented. It is a vortex separator designed to accommodate gas driven two phase flows of gas and liquid. The work presented here is a first of a kind study of this newly invented separator and is meant to determine the minimum inlet gas flow rate necessary for a stable vortex inside the separator for different separator geometries. A dimensional scaling analysis was done to predict this minimum inlet gas flow rate. Experiments were performed on the ground and in conjunction with NASA using their microgravity simulating plane to determine this minimum inlet gas flow rate. The results of the experiments and scaling analysis are compared.

The new design consists of two chambers, a vortex generator and a separation chamber, meant to divide the functions of vortex creation and phase separation. The two phase flow is injected tangentially into the vortex generator causing the inlet linear momentum to be transformed into azimuthal momentum. The two phase mixture in the vortex generator then moves into the separation chamber where the two phases separate due to the density difference between the phases.

The dimensional scaling analysis used the Weber number to predict the minimum rotational velocity of the spinning flow in the separation chamber during a stable vortex. This rotational 
velocity was related to the inlet gas flow rate by the inlet momentum rate. The scaling used the dimensions of each separator tested to predict the minimum inlet gas flow rate needed for a stable vortex.

In all, twelve separators were tested, eleven on the ground and one on the plane. The ground testing was a parametric study varying the sizing of the separator components. The flight experiments kept the separator geometry constant and varied the gravitational field in which the separator operated. In general, the minimum inlet gas flow rate increased with the ratio of separation chamber diameter to vortex generator diameter. This same trend was consistent with the dimensional scaling analysis. Also, the inlet flow rate increases with gravitational acceleration. 


\section{ACKNOWLEDGMENTS}

I would like to thank the support of my committee chair, Dr. Frederick Best, and my committee members, Dr. Karen Vierow and Dr. Gerald Morrison. I would also like to thank Dr. Cable Kurwitz, Dr. Ryoji Oinuma, David Bean, Melissa Ghrist, Ben Larsen, Ngoc Nguyen, and all other past and present members of the Interphase Transport Phenomenon Laboratory that have helped in this research. 


\section{NOMENCLATURE}

Symbol

Bo

$\rho_{f}$

$\rho_{g}$

$R_{I}$

$D_{I}$

$g$

$\sigma$

V

$\omega$

$\mathrm{Fr}$

We

$R_{s c}$

$R_{v g}$

$h_{0}$

$H_{v g}$

$H_{s c}$

$l$

$\mu$
Meaning

$=$ Bond number

$=$ Liquid density

$=$ Gas density

$=$ Inner radius of liquid annulus

$=$ Inner diameter of liquid annulus

$=$ Gravity

$=$ Surface tension of water

$=$ Velocity of the spinning water

$=$ Angular velocity

$=$ Froude number

$=$ Weber number

$=$ Radius of separation chamber

$=$ Radius of vortex generator

$=$ Initial liquid fill level

$=$ Height of vortex generator

$=$ Height of separation chamber

$=$ Viscous sublayer thickness

$=$ Dynamic viscosity of air at room temperature 


$\begin{array}{ll}\dot{p} & =\text { Momentum rate } \\ v_{\text {in }} & =\text { Gas inlet flow velocity } \\ \dot{V}_{\text {in }} & =\text { Gas inlet volumetric flow rate } \\ A_{\text {tube }} & =\text { Cross sectional area of the inlet tube } \\ \mathrm{t} & =\text { time }\end{array}$




\section{TABLE OF CONTENTS}

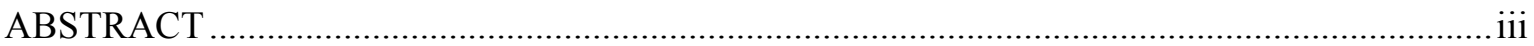

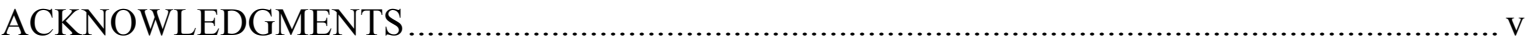

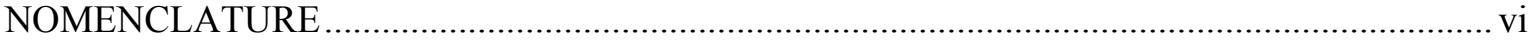

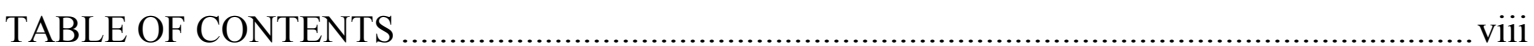

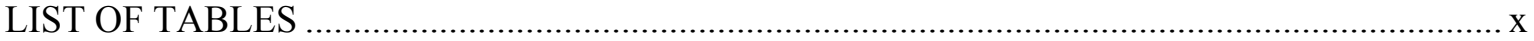

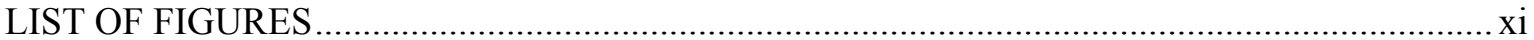

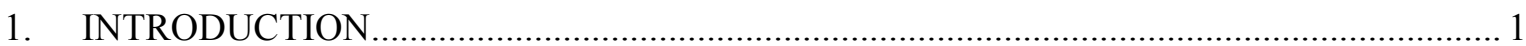

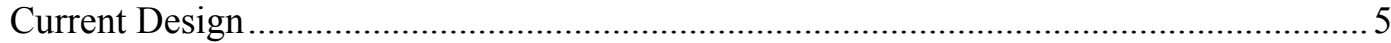

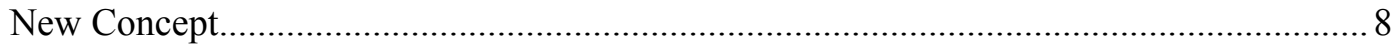

Use of Computational Fluid Dynamics........................................................................ 10



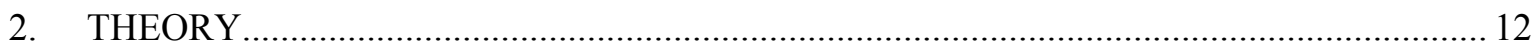

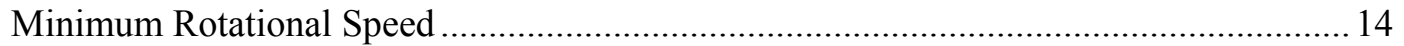

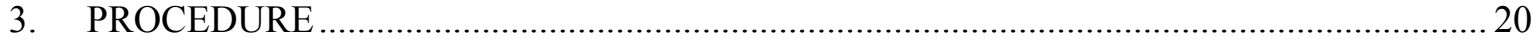

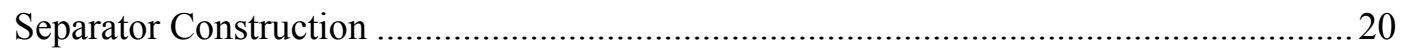

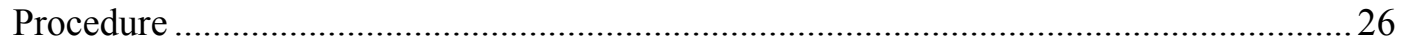

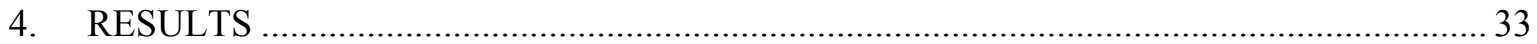

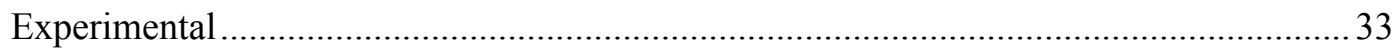

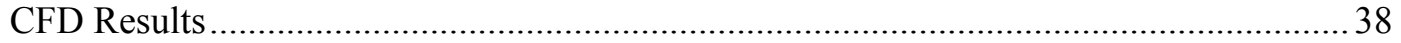

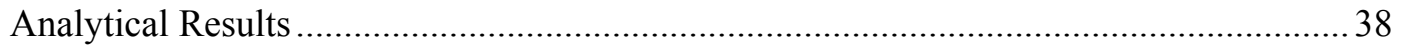






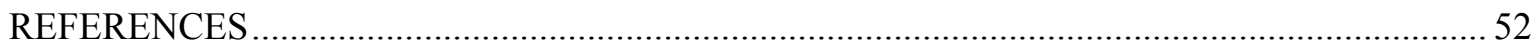

APPENDIX A USE OF COMPUTATIONAL FLUID DYNAMICS ....................................... 54

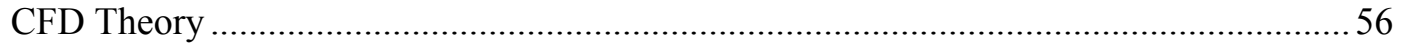

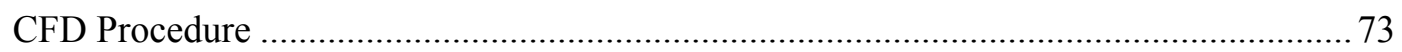

APPENDIX B STATISTICAL ANALYSIS OF FLOW RATE ERROR ..................................... 76

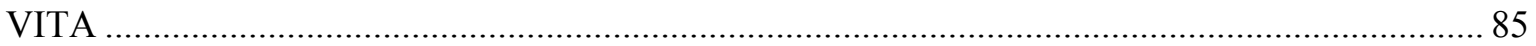




\section{LIST OF TABLES}

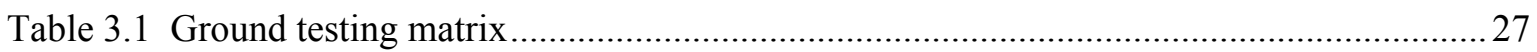

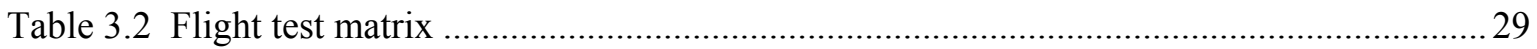

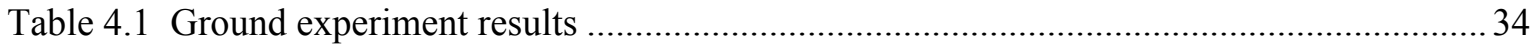

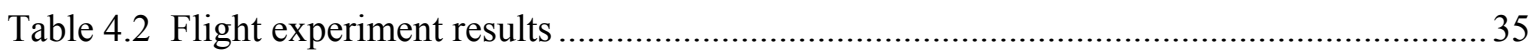

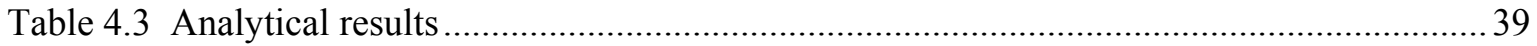

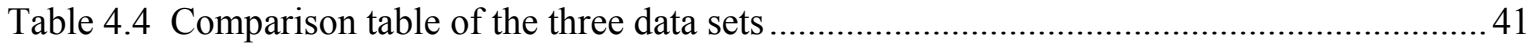

Table 4.5 Comparison of errors for each separation chamber and vortex generator dimension ..... 44

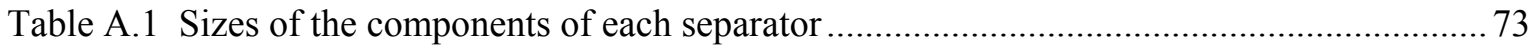

Table A.2 Properties of air and water used in pro-STAR ........................................................ 74

Table B.1 Mean and standard deviation of measurements ......................................................... 76 


\section{LIST OF FIGURES}

Page

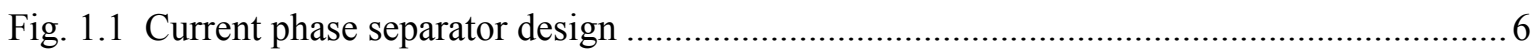

Fig. 1.2 Diagram of the single chamber phase separator in operation......................................... 7

Fig. 1.3 Schematic of the gas driven separator ..................................................................... 9

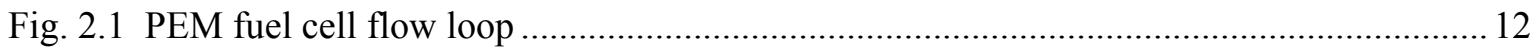

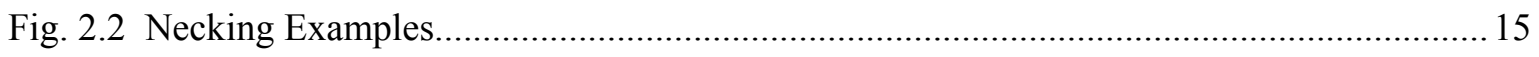



Fig. 3.1 Photograph of a vortex generator under construction ............................................... 21

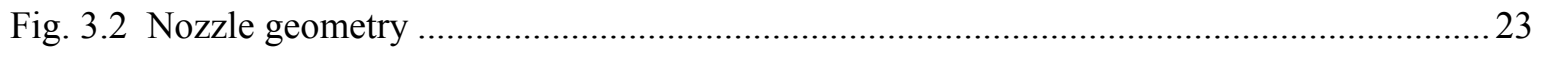

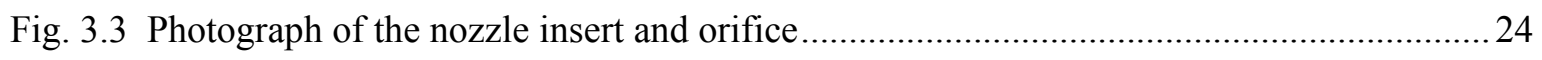

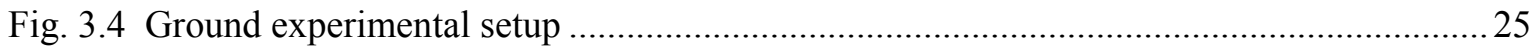

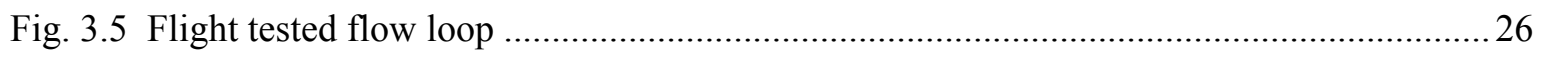

Fig. 3.6 Schematic of the criteria used to judge vortices in ground testing ................................... 30

Fig. 3.7 Flight test vortex acceptability criteria .................................................................... 32



Fig. 4.2 Plot of minimum inlet flow rate for different separator geometries ................................ 37

Fig. 4.3 Analytical results of diameter ratio and inlet gas flow rate .............................................. 40

Fig. 4.4 Comparison of experimental and analytical data for the $0.00030 \mathrm{~m}$ orifice ................... 45

Fig. 4.5 Comparison of experimental and analytical data for the $0.00025 \mathrm{~m}$ orifice .................... 46

Fig. A.1 Representation of two arbitrary neighboring cells ..................................................5 
Fig. A.2 Diagram showing the convention for node labeling for Eq. A.9

Fig. A.3 Schematic of the separator showing the different regions............................................. 62

Fig. A.4 A clipped view of the interior tetrahedral grid for the 1/2"Dx1/2"H vortex generator ..... 66

Fig. A.5 A clipped view of the mesh inside of the 3/4"Dx1"H separation chamber ...................... 68

Fig. A.6 Separation chamber and the relative dimensions of each zone .....................................69

Fig. A.7 Diagram of the first slice of the separation chamber.................................................. 70

Fig. A.8 Diagram of the second section of the separation chamber. ............................................ 71

Fig. A.9 Diagram of the third portion of the separation chamber. .......................................... 71

Fig. A.10 Overall geometry of the separator with the boundary regions identified ....................... 72 


\section{INTRODUCTION}

Swirling motions abound in nature and in the engineering world. Some animals, like the chambered nautilus, exhibit a spiral in its shell which can, remarkably, be described by a logarithmic spiral. Meteorologists are interested in predicting the occurrence and strength of natural vortices such as in tornadoes, hurricanes, and whirlpools. Engineers aim to artificially generate vortices to achieve a useful result such as separating substances of different densities or improving mixing efficiencies in cyclonic furnaces. In the realm of separation, many industries like the coal, petroleum, nuclear, and space industries employ vortex separators. Some of these separators specialize in the separation of dust particles from a gas stream, whereas others focus on separation of liquid and gas.

In an environment where gravity dominates producing body forces, like on earth, vortex separators typically operate at high rotational speeds with the denser substance (usually solid or liquid) falling to the bottom of the device and the less dense substance (usually gas) leaving the top. The chevron and cyclone separators utilized in boiling water reactor (BWR) power plants exemplify this behavior.

In the realm of outer space, two phase flow is commonly found in applications such as air revitalization, water reclamation, thermal management, power management, and solid waste

management. ${ }^{1)}$ All of these areas are vital for the safe and reliable operation of spacecraft, and improvement of phase separation will help these areas and advance space exploration.

This thesis follows the style of Nuclear Science and Technology. 
Challenges in space separators are copious. A separator needs to have high reliability and long operating life, tolerate different flow rates and liquid inventories, operate at low power, and possibly operate in variable g-fields including, but not limited to microgravity. To this end, several devices have been designed and implemented in space and they generally fall into one of two categories: static and rotary separators. Static separators include integral wick, face wick, elbow wick, hydrophilic/hydrophobic, and vortex separators while rotary separators are integral fan, turbine driven, or motor driven separators. ${ }^{2)}$

All of the static separators, except the vortex separator, rely on capillary forces to be the driving mechanism in phase separation. Both the integral wick and face wick separators are implemented in conjunction with condensing heat exchangers. Integral wick separators have the wick on the inside of the heat exchanger to collect water as it condenses. It transports the water to a sump where more wicking material holds the water. A low pressure sink is necessary for water removal and a high bubble point membrane is needed to prevent gas from entering the sink. Similarly, a face wick separator has wicks on the air outlet of a condensing heat exchanger to capture condensate that is swept through by the passing air. This condensate is transferred to a sump and low pressure sink like the integral wick separator. Elbow wick separators take advantage of the momentum of a two phase flow stream by applying a wick material to the outer radius of a tube elbow to collect the liquid that is preferentially thrown to it as the flow moves through the elbow. Again, a sump, low pressure sink, and transfer mechanism similar to the integral wick separator are used. Integral wick separators were designed into the cancelled Manned Orbiting Laboratory and with the Sabatier Reactor. Face wick separators were used on the Lunar Module. Elbow wick separators have been used on the Apollo missions. 
Similar to the face wick separator is the slurper separator. It is also placed at the outlet of a condensing heat exchanger, but the wicking material is replaced with a perforated hydrophilic coated chamber. The perforations in the chamber pull liquid, and some air, through. The resulting flow stream has a lower concentration of liquid. The slurper is placed upstream of a low-flow, motor-driven integral-fan rotary separator, which further separates the flow stream. Use of the slurper is designed to provide energy savings to the downstream rotary separator by acting as a first stage separator. Slurpers have been used aboard the Shuttle, Extravehicular Mobility Unit (EMU), and Spacelab.

In hydrophilic/hydrophobic separators, a screen that is either hydrophilic (if liquid is to be removed) or hydrophobic (if gas is to be removed) is placed in a two phase stream to collect the appropriate phase. If liquid is collected, it is moved to wick filled reservoirs where similar removal techniques to the integral wick separators are used. If gas is collected, the gas will be vented. These types of separators were used on the Apollo missions and inside the EMU.

Rotary separators are different from the previously described separators because they do not involve wicking materials. They operate by rotating a section of duct through which two phase flow travels causing a centrifugal force to act on the flow. The denser liquid is pushed to the wall of the duct and collected in a trough. A Pitot tube connected to a stationary part of the duct removes the water from the trough. Rotary separators are also able to provide transportation of the collected water to a storage tank at a higher pressure than the air stream due to the static pressure that the rotational velocity of the water creates.

There are three classifications of rotary separators based on how the rotation is achieved: turbine, motor-driven, and integral-fan. Turbine rotary separators use blades attached to the rotating drum to extract power from the flow and cause rotation. With this design, no external power is needed, 
although flow speed must be increased to make up for the energy lost to rotating the drum. A motor-driven separator simply uses a motor to rotate the drum and collection trough. Integral-fan rotary separators are constructed with the fan to produce air flow and the drum of the separator on a single shaft so that a single motor can drive both devices. The advantages of this configuration are that it provides a smaller volume and weight than other rotary separators and the capability to adjust fan speed and rotational speed simultaneously. For these reasons, the integral-fan rotary separator is the most widely used rotary separator. Rotary separators in general have been used in the lunar module for fuel cell exhaust separation, the environmental control life support system (ECLSS), and in waste collection systems, among many other applications.

A vortex separator uses the centripetal acceleration of a rotating flow field to create a buoyancy force and causes fluids of dissimilar densities to separate. To achieve rotation, the two phase flow is injected tangentially along the wall of a cylindrical chamber. The gas is orders of magnitude less dense than the liquid, so the buoyancy force causes the gas to move radially inward. The liquid remains on the periphery in a layer where baffling will direct it to an outlet sink. The separator patented by the Interphase Transport Phenomenon (ITP) laboratory at Texas A\&M University (TAMU) is of this type and will be described further below.

Each type of separator offers advantages and disadvantages. In general, rotary separators are recognized as the most versatile classification of separator because they can resist most strongly acceleration transients. However, rotary separators require a power source and the complexity of the moving parts allow a greater opportunity for mechanical failure. Static separator technology requires zero power, but cannot adapt to a large range of flow conditions or accelerations. In addition, static separators involving capillary forces can become less efficient as contaminants in the flow stream obstruct wicks/perforations. The vortex separator developed by TAMU is a 
passive separator that combines the advantages of static and rotary separators. The strong vortex produced in the separator resists the influence of acceleration transients. The liquid layer can accommodate different liquid inventories and inlet flow conditions. The device itself requires zero power and relies only on the momentum of the inlet flow.

\section{Current Design}

The ITP laboratory at Texas A\&M has designed, developed, and patented a vortex phase separator to accomplish microgravity separation. The current design involves a cylindrical chamber into which a two phase flow stream enters tangentially along the wall (see Fig. 1.1 and Fig. 1.2). The phases are separated due to a buoyancy force that is generated by the centripetal acceleration field in the separator and by the density difference between the gas and liquid phases. This design is driven by the flow.

This separator design has benefited from thousands of microgravity parabolas aboard NASA's KC135 and DC-9 aircraft, and has been successfully used in numerous systems. Immense effort has been put into the optimization of this design, and the product of this effort is shown in Fig. 1.2. Two phase flow may enter through one nozzle while another nozzle provides liquid drive flow. With sufficient rotational speed, the injected gas migrates to the center to form a gas core. The gas exits through an outlet tube that protrudes from above into the gas core. A baffle plate at the bottom of the separator provides a barrier to the gas exiting the liquid outlet. The baffle plate also defines the lower limit of water volume with which the separator can operate. 


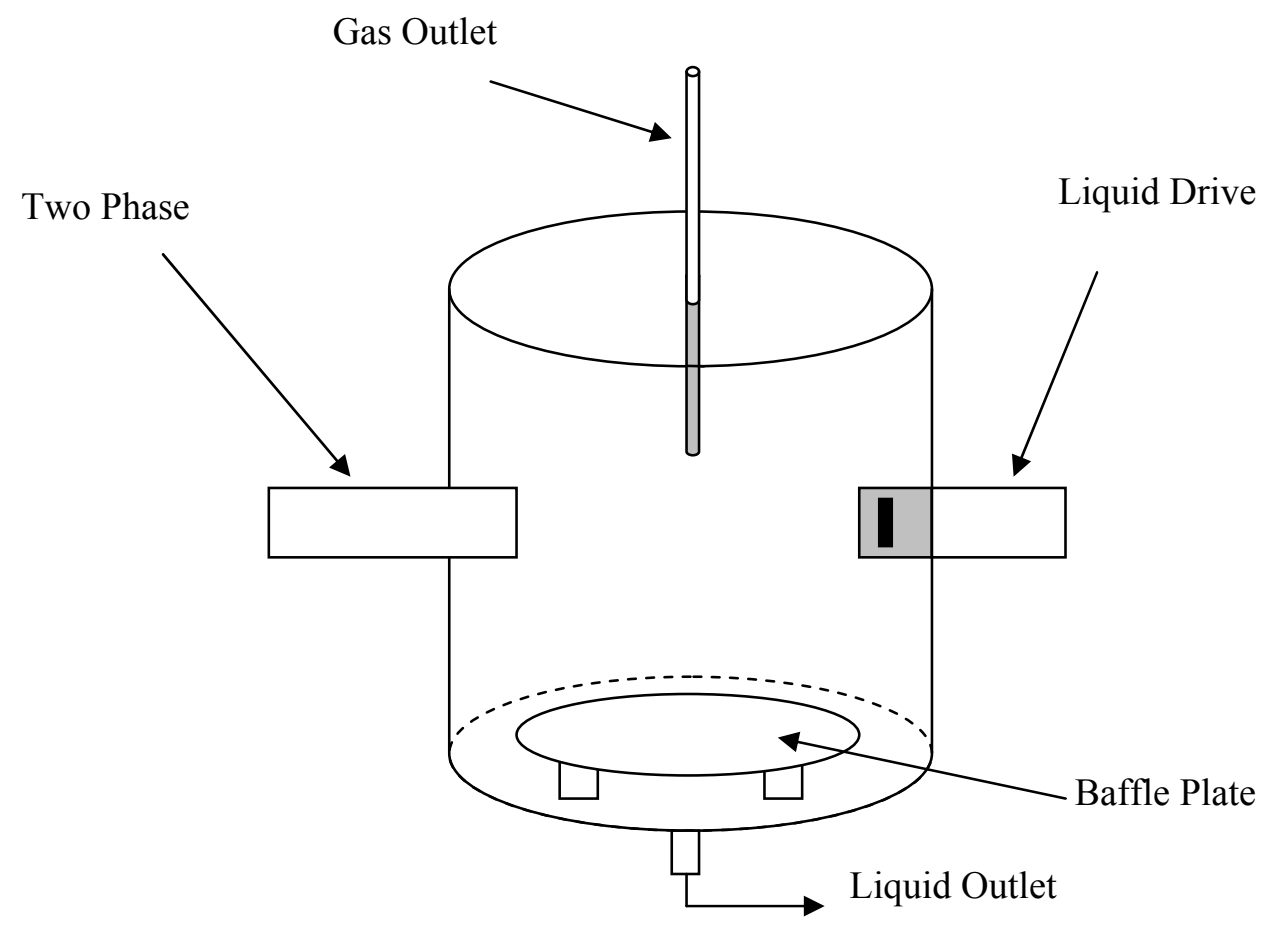

Fig. 1.1 Current phase separator design 




Fig. 1.2 Diagram of the single chamber phase separator in operation

This type of separator may fail by one of two ways: carry under and flooding. The first failure mode is when the liquid layer in the separator is less thick than the gap between the baffle plate and the wall of the separator thus allowing gas to escape the liquid outlet. This mode is also related to inlet bubble transit time. ${ }^{3)}$ If the radial transit time of the bubble is greater than the axial transit time, the bubble will travel beneath the baffle plate and exit the liquid outlet. The radial and axial bubble transit times are inversely proportional to the inlet two phase flow rate, but the radial transit time decreases faster than the axial allowing separation to occur with sufficient inlet flow. ${ }^{3)}$ The second failure mode also concerns liquid volume. With large volumes of liquid, the gas core can become unstable and collapse, allowing liquid to exit the gas outlet. These two modes provide the hydrodynamic operational limits for a given separator. 
In the following sections, a new separator design will be introduced. A better understanding of this new design is desired. The objective of this thesis is to predict the minimum inlet gas flow rate based on the sizes of the separator components necessary for a stable vortex to be achieved in the separator.

\section{New Concept}

The aforementioned separator design works well with systems involving a large liquid volume relative to the gas volume, but degrades as liquid flow decreases. Attempts to use the design from Fig. 1.2 as a gas driven separator were made. This resulted in low separation efficiency due to the inlet gas stream blowing through the liquid buffer layer and entraining droplets in the gas core. This condition is unacceptable. For systems that have high gas flow rates and low liquid flow rates, a dual chambered separator design has been invented. Fig. 1.3 is a schematic of this new design. Major differences between this design and the single chamber design include the absence of a baffle plate, the placement of the gas outlet tube, and the presence of two chambers instead of only one.

In Fig. 1.3, the gas enters the separator in the bottom chamber (the vortex generator) through the nozzle where it is accelerated and creates a rapidly spinning vortex. This vortex travels up to the top chamber (the separation chamber) where it couples with liquid onto which it imparts its rotational momentum. The advantage of this design is that it can operate at relatively low gas flow rates and with low liquid inventories. The gas outlet of the single chamber design is a tube descending from the top of the volume approximately an inch into the gas core.

Another main difference in this design compared with the single volume design is the absence of a baffle plate. In the dual chambered design, the gas core begins at the bottom and extends the entire axial height of the separator. This is possible since the gas outlet tube extends from the vortex 
generator up through the separation chamber. The water outlet is not pictured in Fig. 1.3 since it was not a critical part of the experiment. However, a liquid port was at the top of the separator to inject and drain water.



Fig. 1.3 Schematic of the gas driven separator 
This design was invented for space applications, so its design takes into account the desirable attributes of a space separator previously discussed. It is a passive separator that has no moving parts. Its design can accommodate flow streams that other vortex separators cannot.

Problems arise in the dearth of knowledge about this separator. Because of its new geometry, the types of modeling tools used for the single chamber separator are not always applicable. This gives the inspiration to develop a methodology to characterize the separator and predict its performance based on the inlet flow conditions.

The objective of the work presented is to determine the minimum inlet gas flow rate necessary for a stable vortex to be achieved in the separator. This will be done through ground and flight experiments, and a dimensional scaling technique. The dimensional scaling technique will be discussed in detail in the Theory section. The construction of the separator and experiments performed will be elaborated on in the Procedure section. The Results section will show the experimental results and compare them to the dimensional scaling technique from the Theory section. Finally, the Conclusion section will discuss the main points of emphasis from the whole body of work.

\section{Use of Computational Fluid Dynamics}

There are numerous challenges with modeling a vortex separator including two-phase interfacial shear, compressible flow, multiple dimensions, turbulent conditions, etc. Measurement of some of these quantities is either impossible or prohibitively intrusive. For that reason, it is useful to have a computational tool such as a computational fluid dynamics (CFD) program to evaluate these parameters. For this analysis, the Simulation of Turbulence in Arbitrary Regions (STAR-CD) software package by CD-Adapco was used because of its excellent reputation for handling multiphase systems. Use of CFD analysis is widespread among the two phase flow community 
with examples found specifically in vortex separation. For more details on the use of CFD in two phase flow analysis, refer to Appendix A.

Due to licensing issues, the CFD for the new separator design was not able to be completed, but it provides an opportunity to continue the work presented here.

\section{Experiments}

Several experiments were performed with the newly developed separator, both in a terrestrial environment and in a microgravity environment. The ground experiments consisted of a parametric study that varied the sizing of separator components (vortex generator diameter, separation chamber diameter, and orifice size) and the inlet gas flow rate to find the minimum flow rate to create an acceptable vortex. A similar experiment was conducted in microgravity except that only one separator geometry was tested. The microgravity experiments took advantage of the NASA aircraft and also acquired data at 2-g and lunar gravity conditions. The work presented here will discuss these experiments in more detail and give a comparison of the results with the dimensional scaling technique developed in this thesis.

The following section will describe the development of the model used to predict separator performance. 


\section{THEORY}

The original motivation to invent this new separator was a flow loop like that depicted in Figure 2.1.

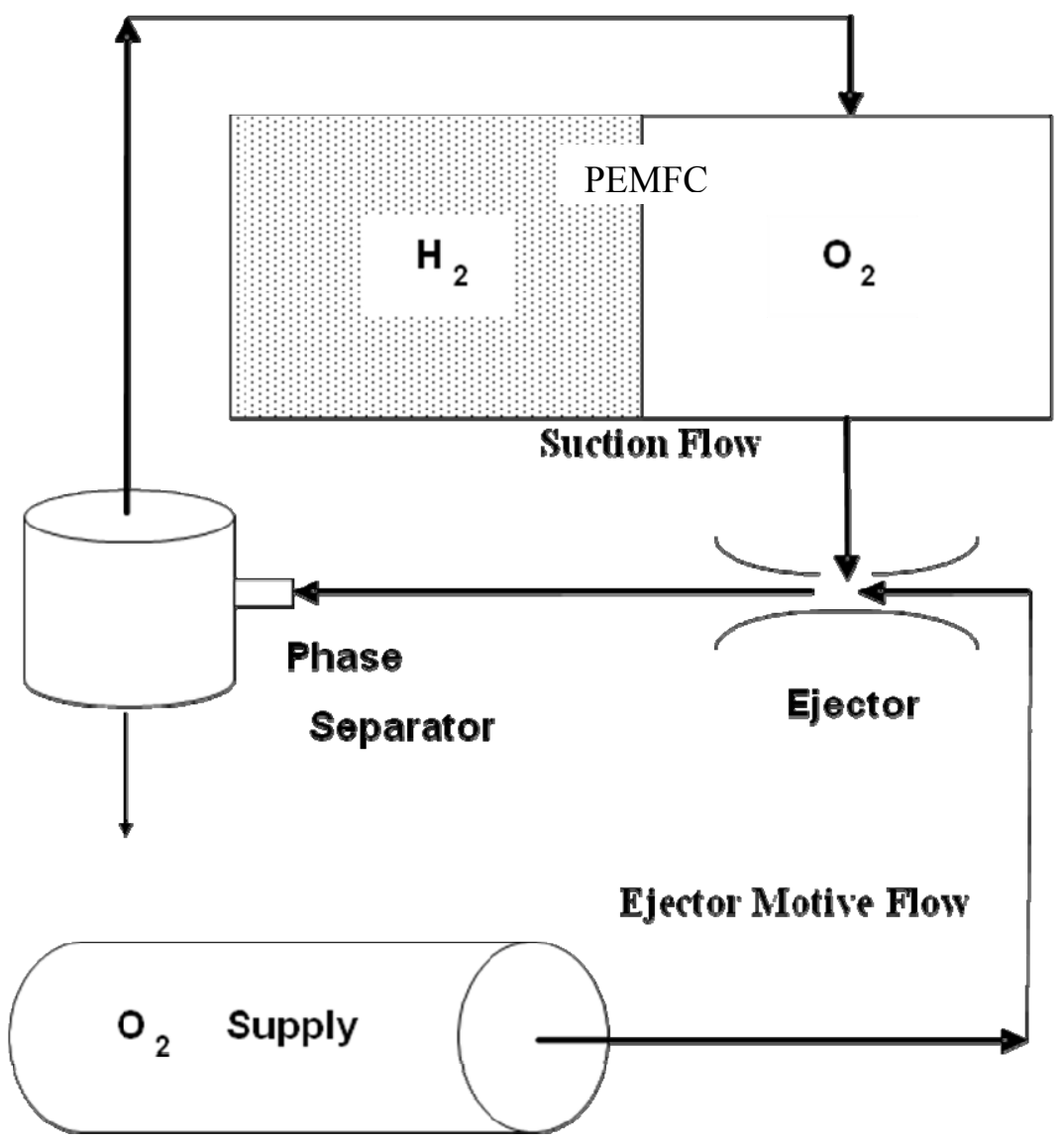

Fig. 2.1 PEM fuel cell flow loop

In this figure, the box labeled "PEMFC" is a proton exchange membrane fuel cell. This fuel cell uses hydrogen gas and oxygen gas to generate electricity. The exhaust from this fuel cell is water from the combined hydrogen and oxygen, and a sweeping flow of oxygen. The oxygen source is a compressed oxygen tank. This tank also provides the motive flow of an ejector whose suction port 
is connected to the outlet of the PEMFC. The resulting flow coming out of the ejector is a gas driven flow with dispersed water droplets in it. The phase separator in this flow loop is designed to separate the oxygen from the water, route the oxygen back to the PEMFC for reuse, and to pass the liquid out to other applications. The liquid driven separator (Fig. 1.1) does not perform well in this type of flow loop. If used for this type of inlet flow, the inlet gas will flow through and interrupt any rotating liquid annulus. This will cause liquid entrainment and compromise separation capability. To circumvent this phenomenon, the separator from Fig. 1.3 was invented.

This separator is designed to divide the functions of vortex generation from phase separation into two different regions. In this way, it aims to eliminate the separation failure due to gas blowing through the liquid annulus. The regions are labeled in Fig. 1.3 as the vortex generator and separation chamber. The vortex generator has a tangential inlet where the two phase flow enters. The linear momentum of the inlet flow is converted into azimuthal momentum through this tangential injection. Inside the vortex generator, the two phases both acquire azimuthal momentum, but they are still mixed together. The mixed flow is forced into the separation chamber while maintaining its azimuthal momentum. Once inside the separation chamber, the liquid and gas will separate due to their density differences, provided there is sufficient rotational speed. The separation chamber behaves very similarly to the liquid driven separator during steady state operation with separation.

The flow loop in Figure 2.1 is proposed for space use. Resources, for example oxygen, are limited in space, so a separator in this flow loop should use the minimum inlet oxygen flow necessary to provide a stable vortex. This is a good rule of thumb for any separator. 


\section{Minimum Rotational Speed}

To develop a model to predict the minimum inlet gas flow rate necessary to create a stable vortex in the separation chamber, the minimum rotational speed for separation needs to be determined. Past experiments and analysis of the separator of Fig. 1.1 utilized the Weber number $(W e)$, Eq. (2.4).

The definition of the Weber number is the ratio of inertial forces to surface tension forces. For the separator of Fig. 1.1, this translates to a minimum rotational speed for a solid gas core. If the rotational speed inside the separator is not high enough during steady state, the inertia of the flow will not be high enough to dominate the surface tension forces. This will cause the surface tension forces to attempt to alter the gas core shape to one of reduced surface energy. This phenomenon is known as necking and is depicted in Fig. 2.2. These are just simple examples of the ways that necking can look. In each case, the rotational speed of the flow is not sufficient to maintain a solid gas core. The leftmost picture has the gas core being collapsed into a single bubble. The middle and rightmost pictures show a gas core that has liquid bridging across it interrupting the gas core and compromising separation. The difference between these two pictures is the liquid inventory in each. The middle picture has more liquid than the rightmost picture. A larger liquid inventory translates into a higher rotational speed necessary to prevent necking. The Weber number corresponding to the minimum rotational speed for a stable gas core for the rightmost picture would not be a high enough Weber number for the middle picture. 

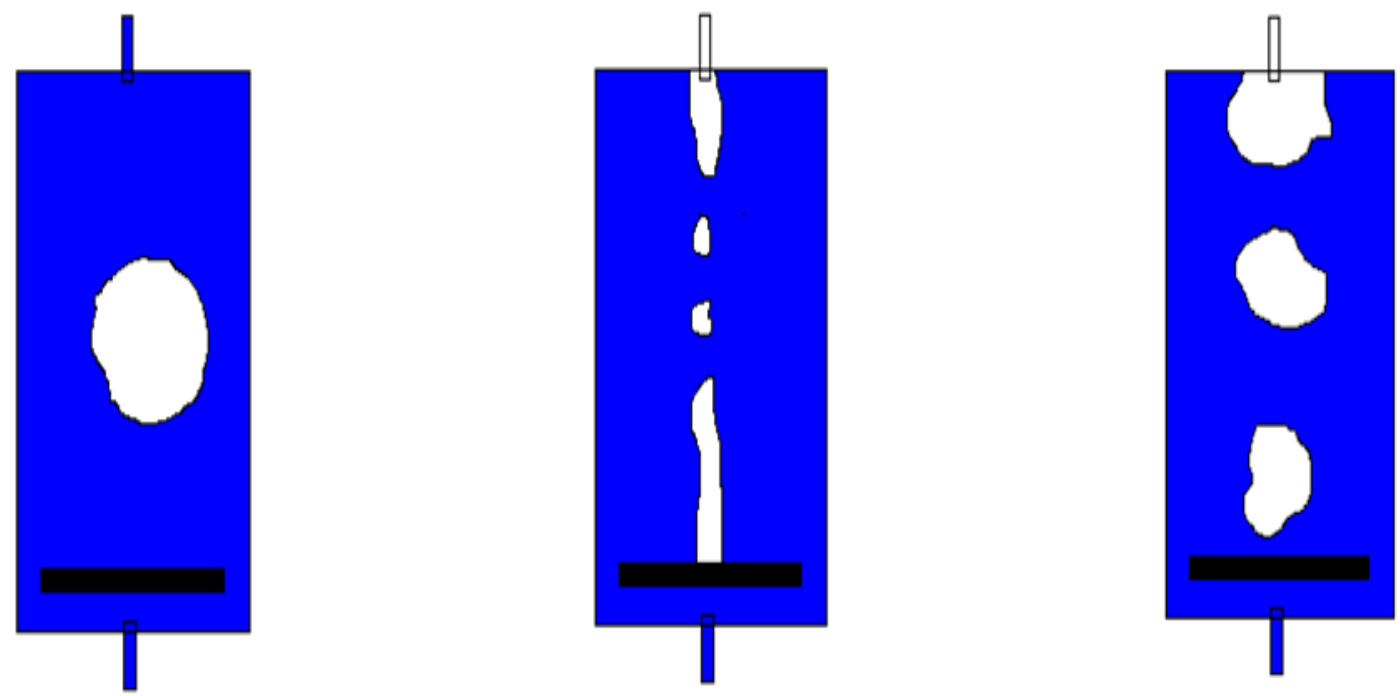

Fig. 2.2 Necking Examples

During steady state operation with separation occurring in the newly invented separator, the separation chamber behaves similarly to the liquid driven separator. For this reason, it is assumed that use of the Weber number is acceptable to predict the minimum rotational velocity to produce a stable vortex. The use of the Weber number in the gas driven separator is only applicable in the separation chamber. It does not describe the physics in the vortex generator since the two phase mixture in it is not experiencing separation.

To determine an equation for $W e$ the Bond number $(B o)$ and the Froude number $(F r)$ were used. ${ }^{4)}$ Equations (2.1) and (2.2) show Bo and Fr respectively. The Bond number is the ratio of acceleration forces to surface tension forces. For the separator, this number would be relevant during flow rate changes to ensure any acceleration would not allow surface tension forces to cause necking. The Froude number is the ratio of inertial forces to acceleration forces and would be used 
to predict if the inertial forces are too low due to the acceleration forces being too high. Assuming that the spinning water rotates as a solid body, Eq. (2.3) was used to relate the velocity to the angular velocity. Multiplying $B o$ and $F r$ gives an equation for $W e$. After some simplification, Eq. (2.4) is the resulting Weber number.

$$
\begin{gathered}
\text { Bo }=\frac{\text { acceleration forces }}{\text { surfacetension forces }}=\frac{\left(\rho_{f}-\rho_{g}\right) R_{I}^{2} g}{\sigma} \\
F r=\frac{\text { inertial forces }}{\text { acceleration forces }}=\frac{V^{2}}{g D_{I}} \\
V=R_{I} \omega \\
\text { We }=\frac{\text { inertial forces }}{\text { surfacetension forces }}=\frac{\left(\rho_{f}-\rho_{g}\right) R_{I}^{3} \omega^{2}}{2 \sigma}
\end{gathered}
$$

where $\rho_{f}$ and $\rho_{g}$ are the liquid and gas densities, $g$ is gravity, $\sigma$ is the surface tension of water, $V$ is the velocity of the spinning water, and $\omega$ is the angular velocity.

$D_{I}$ and $R_{I}$ are the inner diameter and radius respectively of the liquid annulus developed in the separation chamber. This was determined by knowing that the initial fill level during each experiment was one quarter of the total separation chamber height. Each separation chamber was the same height $(0.0254 \mathrm{~m})$, so the same initial fill level was used for each separator. Equation (2.5) was used to calculate $R_{I}$. Bean et al. ${ }^{4)}$ showed that maintaining $W e$ above 100 is necessary for a stable vortex. Applying this result and Eq. (2.5) in Eq. (2.4), Eq. (2.6) is used to determine the minimum rotational speed for each separator. 


$$
\begin{gathered}
R_{I}=\sqrt{\frac{R_{s c}^{2} h_{0}+R_{v g}^{2} H_{v g}}{H_{s c}}} \\
\omega=\sqrt{\frac{200 \cdot \sigma}{R_{I}^{3}\left(\rho_{f}-\rho_{g}\right)}}
\end{gathered}
$$

To support the use of using the Weber number equal to 100, Fig. 2.3 is given. In the figure, the dimensionless diameter ratio of the interface diameter, $D_{i}$, to the separator diameter, $D$, is on the ordinate. The rotational speed in radians per second is given on the abscissa. The diamonds represent a cylindrical gas core, the hollow squares an intermediate gas core, and the crosses a necking gas core. There is one $\mathrm{x}$ on the figure which corresponds to an error. There are three Weber number lines on the plot. The solid line is for $W e=1$, the coarse dashed line is for $W e=10$, and the fine dashed line is for $W e=100$. The points below the $W e=100$ mainly consist of crosses with a few hollow squares. The points above $W e=100$ are mainly diamonds and some squares. Near $W e=100$ are mainly squares. This indicates that $W e=100$ marks a transition from necking into a cylindrical gas core. This figure was created for a liquid driven separator, but since the separation chamber of the gas driven separator behaves like the liquid driven separator during steady state operation, then it is applied for the gas driven separator. 


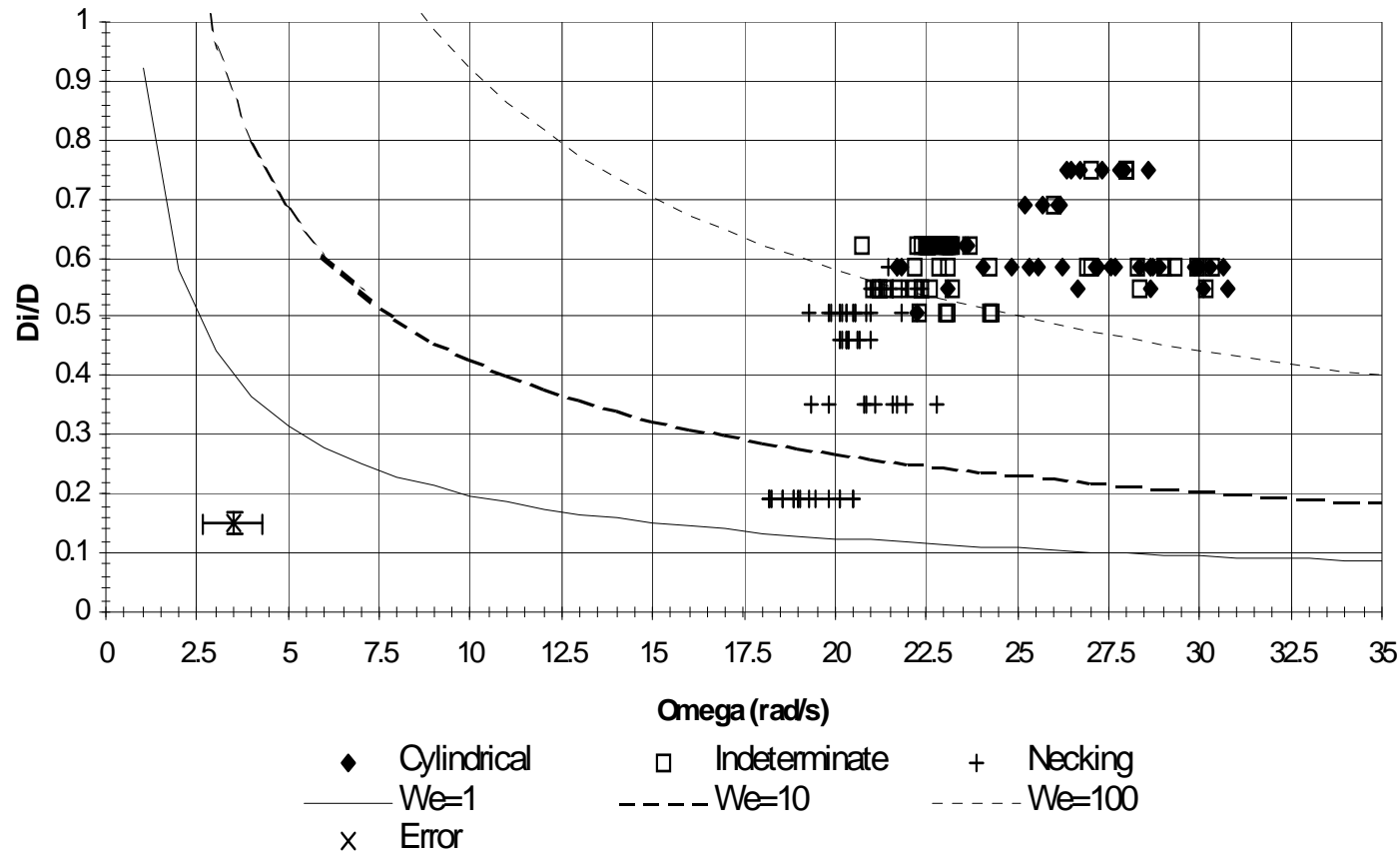

Fig. 2.3 Weber number justification

Knowing the necessary $\omega$, the inlet gas flow rate needed to produce this $\omega$ is desired. Ellis ${ }^{3)}$

showed that for the separator of Fig. 1.1 the inlet momentum rate, $\dot{p}$, correlates to $\omega$ by Eq. (2.7).

$$
\omega=\frac{l}{\mu} \frac{\dot{p}}{8 \pi^{2} R_{I}^{3}}
$$

where $l$ is the viscous sublayer thickness, and $\mu$ is the dynamic viscosity of air at room temperature.

Here, $\dot{p}$ is given by Eq. (2.8).

$$
\dot{p}=\rho_{g} v_{\text {in }}^{2} A_{\text {tube }}
$$


The inlet velocity, $v_{i n}$, is related to the inlet volumetric flow rate, $\dot{V}_{i n}$, by Eq. (2.9), where $A_{\text {tube }}$ is the cross sectional area of the inlet tube.

$$
v_{\text {in }}=\frac{\dot{V}_{\text {in }}}{A_{\text {tube }}}
$$

Combining Eq. (2.7), (2.8), and (2.9) and solving for $\dot{V}_{\text {in }}$ :

$$
\dot{V}_{\text {in }}=\sqrt{\frac{8 \pi^{2} \omega \mu R_{I}^{3} A_{\text {tube }}}{l \rho_{g}}}
$$

An estimated value of $0.0005 \mathrm{~m}$ was used in all cases for the viscous sublayer thickness. With Eq. (2.10), the gas inlet volumetric flow rate needed for a stable vortex was determined. Equation (2.10) is the equation used for comparison to the experimental results.

For details on the CFD model development, refer to Appendix A. 


\section{PROCEDURE}

There are three sets of evaluations of the separator: ground testing, microgravity testing, and computational testing. The procedure for each is described below. Eleven different configurations of vortex generator, separation chamber, and orifice diameter were ground tested. The same eleven geometries were planned to be simulated with CFD, but licensing issues of the software precluded the completion of all of the tests. CFD details are in Appendix A. Only one geometry was tested in microgravity. Each test used single phase gas at the inlet. Liquid was injected as needed between flow rate increments to a fill level of approximately half the axial height of the separation chamber. This condition was chosen to simplify the test setup and to allow ease of comparison between the experimental and CFD results.

\section{Separator Construction}

The goal of the experiments was to test different combinations of vortex generator, separation chamber, and orifice diameter. Because of this, the construction of the separator facilitated the interchangeability of these components. The vortex generators and separation chambers were all made of $0.0635 \mathrm{mx} 0.0635 \mathrm{mx} 0.02540 \mathrm{~m}$ pieces of clear acrylic. The separation chambers and vortex generators were matched together with an o-ring or a rubber gasket between them to provide an air seal. Four bolts held the two pieces together.

To manufacture the vortex generator, the center of a piece of acrylic was located (refer to Fig. 3.1). A whole whose depth matched the diameter of the vortex generator was drilled into the center of the acrylic. A flat bottomed drill bit was used to make the bottom of the vortex generator flat. The tunnel leading into the vortex generator was drilled such that its center was at the axial middle of the vortex generator and tangential to the edge of the vortex generator. Two holes were actually drilled for this. One smaller diameter hole was drilled which penetrated the vortex generator 
allowing the orifice to match up with the vortex generator. Another larger hole was drilled partially through the first hole. This was for the nozzle insert to screw into. Not shown in Fig. 3.1 are the four bolt holes in each corner of the acrylic for the bolts that hold the vortex generator and separation chamber together. Also not shown is the hole made for the gas outlet tube. In each vortex generator, the gas outlet tube was a stainless steel pipe with an outer diameter of 0.003175 m. A $0.003572 \mathrm{~m}$ diameter hole was drilled in the radial center of the vortex generator all the way through the acrylic block. Another larger hole was made concentric with the outlet tube hole to accommodate an o-ring fitting that mated with the outside of the vortex generator.



Fig. 3.1 Photograph of a vortex generator under construction 
The separation chamber used a similar manufacturing technique as the vortex generator. All of the separation chambers had the same height of $0.0254 \mathrm{~m}$, which was the height of the acrylic blocks. Therefore, a hole was drilled in the center of the block all the way through. There were also bolt holes in the corners of the block to match up with the vortex generator block. This was all of the machining for the separation chamber used in the ground testing, but the flight tested separator included a liquid port in the axial middle of the separation chamber, in line with the center axis of the separation chamber.

The nozzle for most of the tests was a two piece insert consisting of a brass spacer and a brass orifice. The spacer was a slice of brass rod with a through-hole drilled in the axial center threaded with 10-32 thread. The outer circumference of the spacer was threaded with 3/8"-24 threads. The orifices were purchased from Beswick Engineering. This geometry is illustrated in Fig. 3.2. A photograph of the nozzle insert and orifice is in Fig. 3.3. A change was made in this design for later tests because of gas leakage around the outside threads of the nozzle insert that occurred at large inlet pressures. The modified design eliminated the brass spacer and screwed the orifice directly into the fitting which connected to the vortex generator. This design caused the outlet of the orifice not to directly penetrate the vortex generator causing a small gap on the order of millimeters between the orifice outlet and the vortex generator. It is not believed that this gap had a significant effect on separation ability. 
Spacer, 3/8-

24 thread

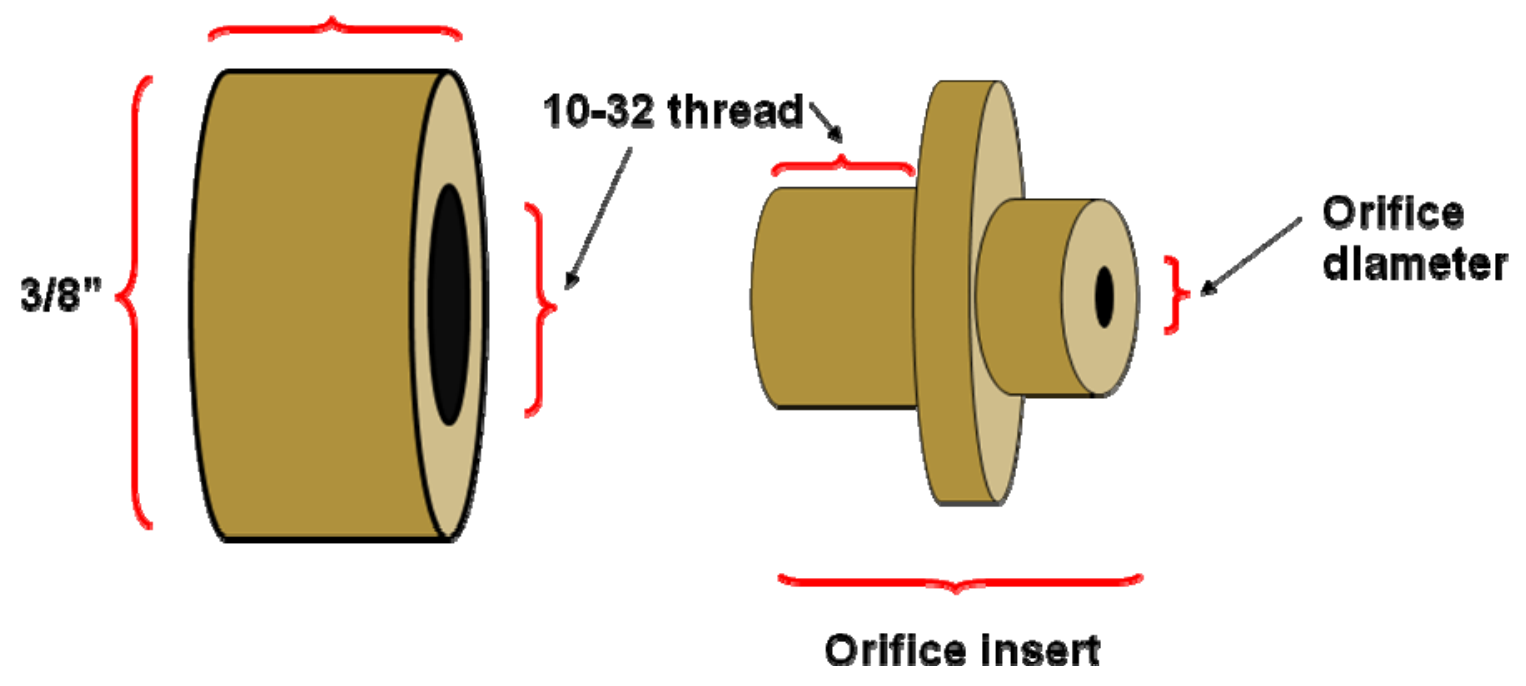

Fig. 3.2 Nozzle geometry 


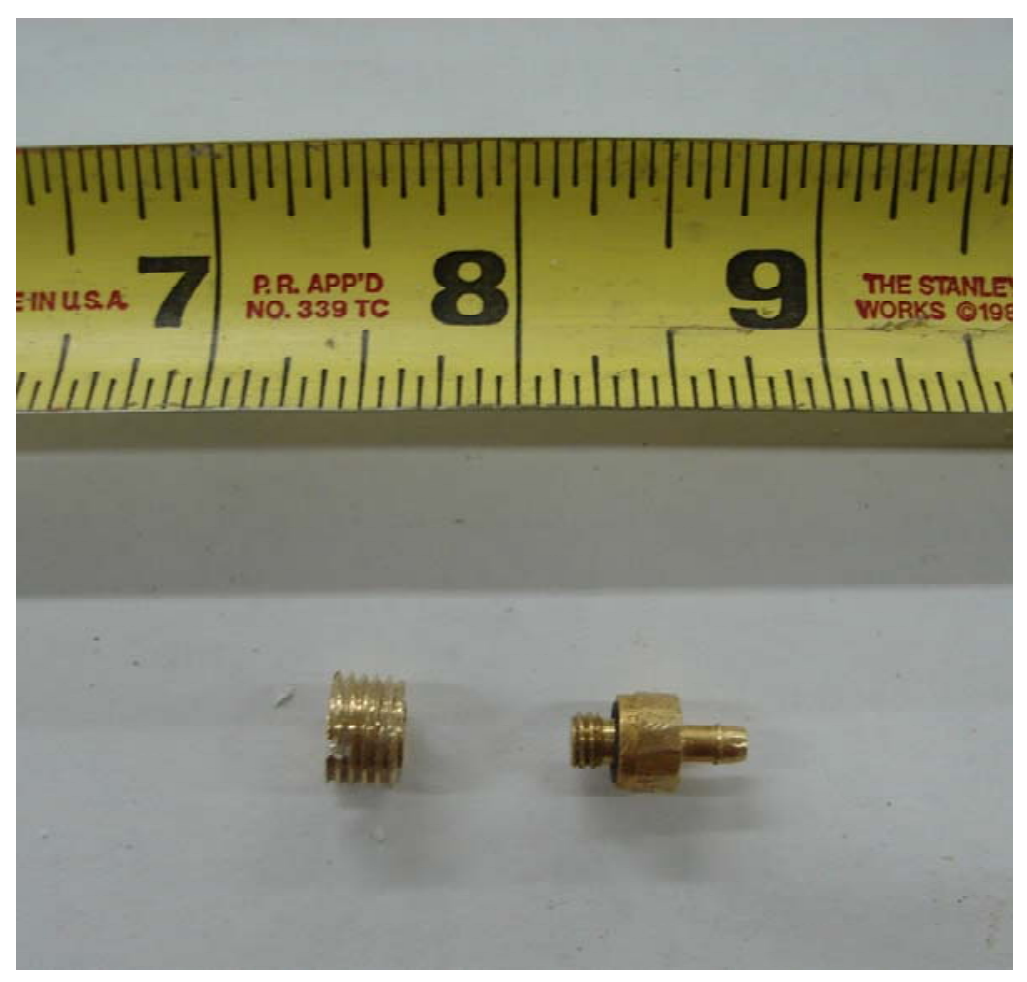

Fig. 3.3 Photograph of the nozzle insert and orifice

Since the separation chamber and vortex generators were made from separate pieces of acrylic, there was the task of joining them together such that they were concentric. The concentricity of the pieces varied and any asymmetry caused the azimuthal momentum developed in the vortex generator to be less coupled to the separation chamber.

The same general construction was used for the flight tested separator, except the nozzle did not include the orifice. The nozzle was built from brass with an opening of $0.003175 \mathrm{~m} \times 0.000762 \mathrm{~m}$. There was also a dedicated liquid injection port on the flight tested separator that was omitted from the ground tests. 
The ground test setup is shown in Fig. 3.4. The inlet gas flow was controlled using a metering valve and measured with a digital flow meter. Flexible tubing with an inner diameter of $0.00635 \mathrm{~m}$ was used for the gas leading into the separator. Gas traveled through the orifice and into the vortex generator. A mechanical pressure gauge was used to read the separator inlet pressure. The inside of the separator was at atmospheric conditions. The tube protruding from the top of the separator in Fig. 3.4 was where liquid was injected. This tubing, as well as the gas outlet tube, was stainless steel with an outer diameter of $0.003175 \mathrm{~m}$. This port was closed during tests, so the only place open to the atmosphere was the gas outlet tube.

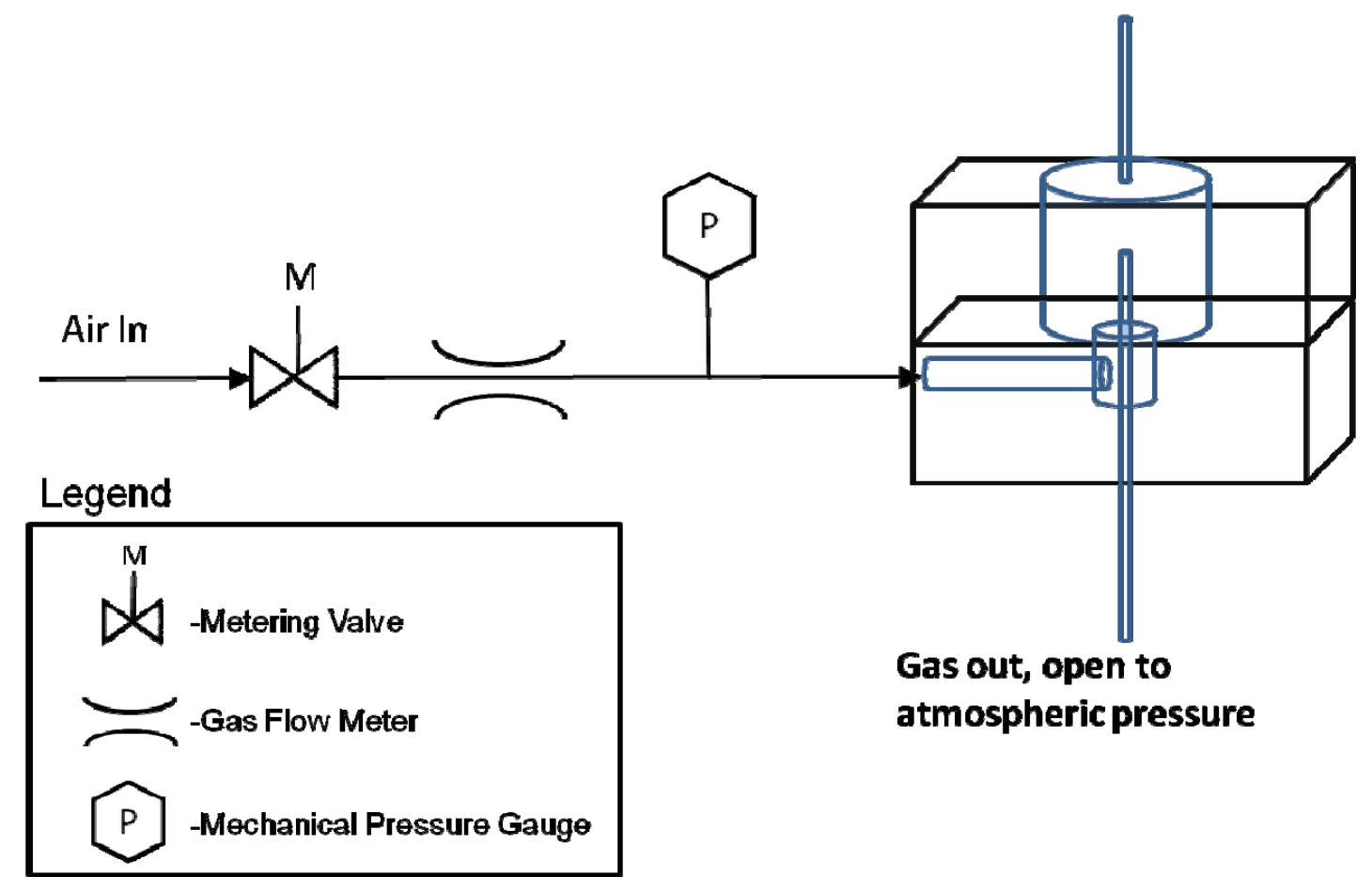

Fig. 3.4 Ground experimental setup

The flow loop for the flight tested separator was very similar to the ground tests. It is shown in Fig. $3.5^{5)}$. It used the same sizes of tubing as the ground testing. A differential pressure gauge 
(DP) was included between the liquid injection port and the gas core region to measure the pressure differential across the liquid layer.

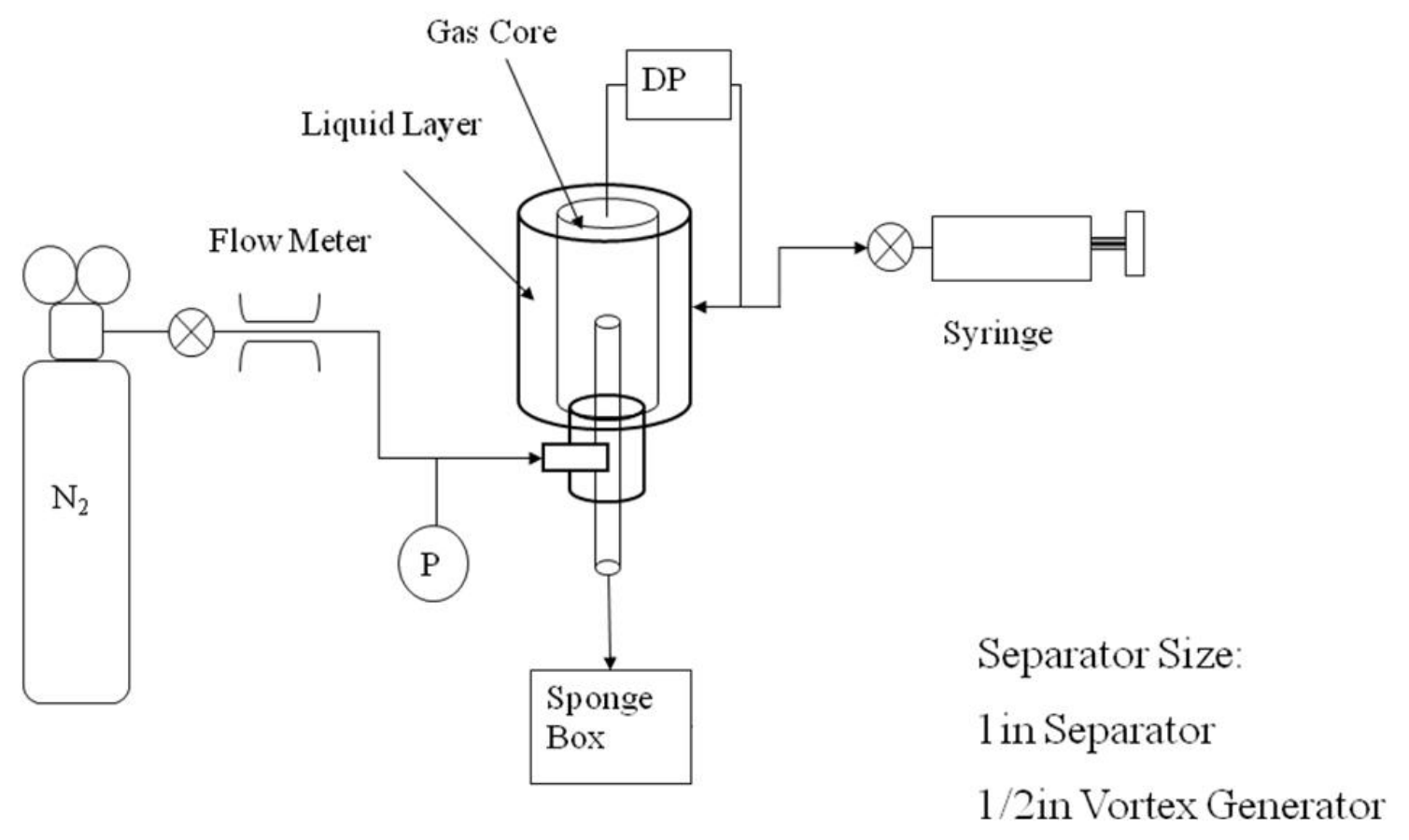

Fig. 3.5 Flight tested flow loop

\section{Procedure}

For each combination of separation chamber diameter, vortex generator diameter, and orifice diameter, the following testing procedure was performed. The inlet gas flow rate was increased by 1 SLPM increments from 1-10 SLPM and then back down from 10-1 SLPM. At each flow rate increment, the vortex in the separation chamber was allowed to stabilize and evaluated for quality. After each flow rate, if significant water exited, the gas flow was stopped and the water level restored to the original fill height. In the flight experiments, water was added until approximately 
$10 \mathrm{ml}$ of water was in the flow loop. This corresponded to a fill level of about one quarter of the axial height of the separation chamber. The next flow rate was then tested. Also, the flow rate increments were not 1 SLPM but were determined instead by inlet pressure increments ranging from 4 psig up to 16 psig. A sample ground test matrix is given in Table 3.1 and a sample flight test matrix in Table 3.2.

Table 3.1 Ground testing matrix

Orifice Size:

Separation Chamber Diameter:

Vortex Generator Diameter

Initial Water Fill

\begin{tabular}{c|c|c|l} 
Gas Inlet Flow Rate (LPM) & $\begin{array}{c}\text { Inlet Pressure } \\
(\mathrm{psig})\end{array}$ & $\begin{array}{c}\text { Good Vortex? } \\
(\mathrm{Y} / \mathrm{N})\end{array}$ & Comments \\
\hline 1 & & & \\
\hline 2 & & & \\
\hline 3 & & & \\
\hline 4 & & & \\
\hline
\end{tabular}


Table 3.1 Continued

\begin{tabular}{|c|c|c|c|}
\hline Gas Inlet Flow Rate (LPM) & $\begin{array}{c}\text { Inlet Pressure } \\
\text { (psig) }\end{array}$ & $\begin{array}{l}\text { Good Vortex? } \\
\qquad(\mathrm{Y} / \mathrm{N})\end{array}$ & Comments \\
\hline 5 & & & \\
\hline 6 & & & \\
\hline 7 & & & \\
\hline 8 & & & \\
\hline 9 & & & \\
\hline 10 & & & \\
\hline 10 & & & \\
\hline 9 & & & \\
\hline 8 & & & \\
\hline 7 & & & \\
\hline 6 & & & \\
\hline 5 & & & \\
\hline 4 & & & \\
\hline 3 & & & \\
\hline 2 & & & \\
\hline
\end{tabular}


Table 3.1 Continued

\begin{tabular}{|c|c|c|c|}
\hline Gas Inlet Flow Rate (LPM) & $\begin{array}{c}\text { Inlet Pressure } \\
\text { (psig) }\end{array}$ & $\begin{array}{l}\text { Good Vortex? } \\
\qquad(\mathrm{Y} / \mathrm{N})\end{array}$ & Comments \\
\hline 1 & & & \\
\hline
\end{tabular}

Table 3.2 Flight test matrix

\begin{tabular}{|c|c|c|c|}
\hline Parabola Number & Inlet Pressure (psig) & Good vortex in 0 -g? & Good vortex in 2-g? \\
\hline 1 & & & \\
\hline 2 & & & \\
\hline 3 & & & \\
\hline 4 & & & \\
\hline 5 & & & \\
\hline 6 & & & \\
\hline 7 & & & \\
\hline 8 & & & \\
\hline 9 & & & \\
\hline 10 & & & \\
\hline
\end{tabular}


Fig. 3.6 shows the criteria used to determine the quality of a vortex of a ground test point. These are the general guidelines used for this determination:

- If any significant amount or constant liquid leaves the gas outlet, the vortex is unacceptable.

- If the liquid in the separation chamber is not spinning as a body, the vortex is unacceptable. Parabolic vortices are acceptable as long as the liquid is spinning as a body.

- If there is significant liquid in the vortex generator that has not been driven into the separation chamber, the vortex is unacceptable. A very small amount of liquid in the vortex generator was allowed for an acceptable vortex provided that the other conditions were met.

\section{Bad Vortex}

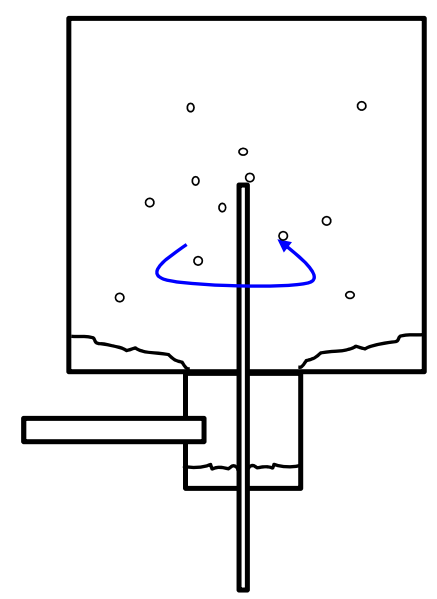

Good Vortex

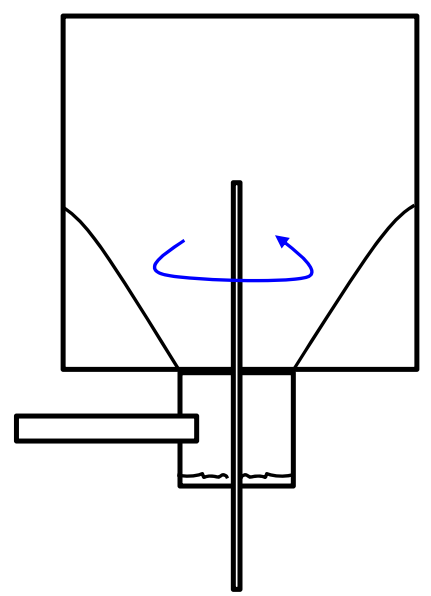

Good Vortex

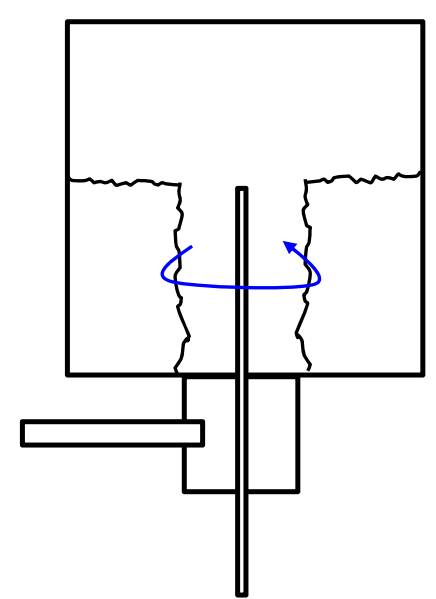

Fig. 3.6 Schematic of the criteria used to judge vortices in ground testing 
In each case in Fig. 3.6, the blue arrow indicates the rotational direction of the gas vortex. The leftmost picture shows a vortex that does not have the liquid spinning as a body, and significant liquid is remaining in the vortex generator. When this type of vortex was observed, the gas typically sheared liquid off from the liquid layer formed inside of the separation chamber. This liquid would get entrained in the gas and exit through the gas outlet. The middle picture shows the lower limit of an acceptable vortex. The liquid is spinning as a rigid body with a parabolic shape. A small amount of liquid remains in the vortex generator, and zero liquid is leaving the gas outlet. The rightmost picture shows the best vortex that was observed. The gas/liquid interface is vertical as opposed to parabolic, all the liquid is expelled from the vortex generator, and there is no liquid exiting the gas outlet. The results for each experiment are presented and discussed in the next section.

The flight testing criteria is very similar to the ground testing criteria. The difference is that there is not a parabolic type of vortex when under microgravity. This is shown in Fig. 3.7. The left picture shows a case with insufficient inlet flow to generate a stable vortex, so the liquid is blown around with a random distribution. The right picture shows the case when sufficient inlet flow exists, and a gas core is formed with a liquid annulus around it. 


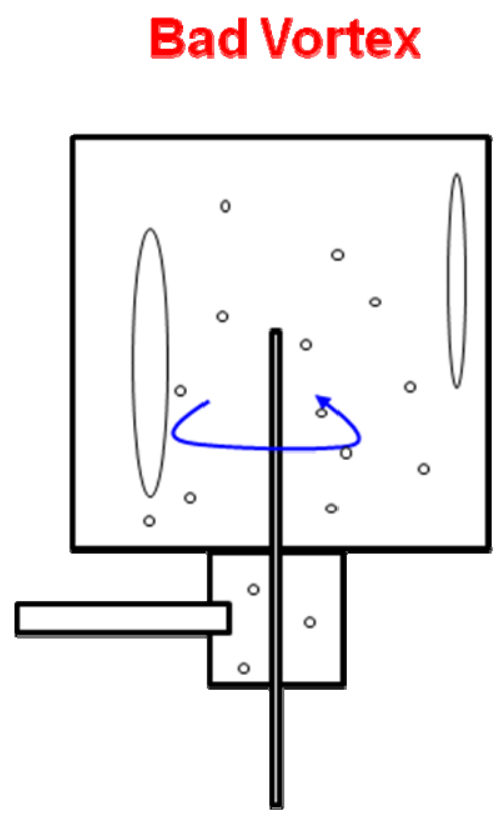

\section{Good Vortex}

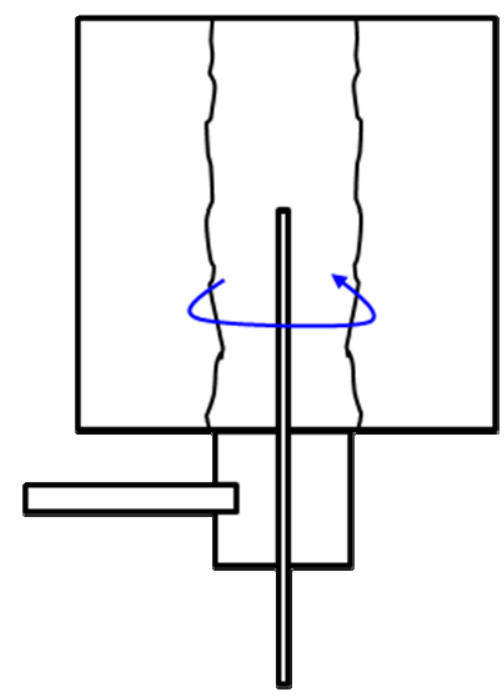

Fig. 3.7 Flight test vortex acceptability criteria 


\section{RESULTS}

The results of the experiments described in the procedure section are given in this section. The analytical calculations described in the Theory section, Eq.(2.10), are also provided. Each set of data will be presented in its own section and a comparison of the data will be made at the end.

\section{Experimental}

As described in the procedure section, eleven different separators were ground tested, plus one in microgravity. The results are given in

Table 4.1 and Table 4.2. Although the flow rate increments used were 1 SLPM, values like 3.5 SLPM were obtained by taking the average between flow rates of 3 and 4 SLPM. This occurred when, during the flow rate ascension from 1-10 SLPM, an acceptable vortex occurred at 3 SLPM, and during the descent, at 4 SLPM.

The smallest acceptable flow rate observed was 2 SLPM for separators 3 and 10. The largest was 8 SLPM for separator 11. This large flow rate occurred with the smallest vortex generator and separation chamber. For the same separator geometry but with the larger orifice, the flow rate was 3.5 SLPM. The other separators with identical geometries, except orifice size, only showed a maximum absolute difference of 1 SLPM. This suggests that the 8 SLPM measurement could be an erroneous data point. There are four geometries that are identical in the separation chamber and vortex generator diameters and differ only in the orifice size. Namely, separators 1, 2, 3, and 6 match $8,9,10$, and 11 . In separators 1 and 8 , the larger orifice size allowed for a larger inlet flow rate with a difference of 1 SLPM. Separators 2 and 9, however, allowed for a lower inlet flow rate for the larger orifice, also differing by 1 SLPM. Separators 3 and 10 used the same flow rate. Finally, separators 6 and 11 showed a larger flow rate for the smaller orifice, with a difference of 4.5 SLPM. It is difficult to decide if a smaller orifice allows for a lower flow rate or not. 
Physically this should be true since a smaller orifice will accelerate the flow more than a larger one. Based on this, more tests should be conducted to elucidate this relationship.

Table 4.1 Ground experiment results

\begin{tabular}{|c|c|c|c|c|c|}
\hline Orifice Diameter (m) & $\begin{array}{l}\text { Separator } \\
\text { Number }\end{array}$ & $\begin{array}{c}\text { Vortex Generator } \\
\text { Diameter (m) }\end{array}$ & \begin{tabular}{|} 
Separation \\
Chamber Diameter \\
(m)
\end{tabular} & $\begin{array}{c}\text { Separation } \\
\text { Chamber to } \\
\text { Vortex } \\
\text { Generator } \\
\text { Diameter } \\
\text { Ratio }\end{array}$ & $\begin{array}{c}\text { Minimum Gas } \\
\text { Flow Rate for Good } \\
\text { Vortex (SLPM) }\end{array}$ \\
\hline \multirow[t]{7}{*}{0.00030} & 1 & 0.01111 & 0.02540 & 2.29 & 5 \\
\hline & 2 & 0.01111 & 0.02223 & 2.00 & 3 \\
\hline & 3 & 0.01111 & 0.01905 & 1.71 & 2 \\
\hline & 4 & 0.01270 & 0.02223 & 1.75 & 3 \\
\hline & 5 & 0.01270 & 0.01905 & 1.50 & 3.5 \\
\hline & 6 & 0.00953 & 0.01905 & 2.00 & 3.5 \\
\hline & 7 & 0.00953 & 0.01588 & 1.67 & 3 \\
\hline \multirow[t]{4}{*}{0.00025} & 8 & 0.01111 & 0.02540 & 2.29 & 4 \\
\hline & 9 & 0.01111 & 0.02223 & 2.00 & 4 \\
\hline & 10 & 0.01111 & 0.01905 & 1.71 & 2 \\
\hline & 11 & 0.00953 & 0.01905 & 2.00 & 8 \\
\hline
\end{tabular}


Table 4.2 Flight experiment results

\begin{tabular}{|c|c|c|c|c|c|}
\hline Orifice Diameter (m) & $\begin{array}{c}\text { Separator } \\
\text { Number }\end{array}$ & $\begin{array}{c}\text { Vortex Generator } \\
\text { Diameter (m) }\end{array}$ & $\begin{array}{c}\text { Separation } \\
\text { Chamber Diameter } \\
\text { (m) }\end{array}$ & $\begin{array}{c}\text { Separation } \\
\text { Chamber to } \\
\text { Vortex } \\
\text { Generator } \\
\text { Diameter } \\
\text { Ratio }\end{array}$ & $\begin{array}{c}\text { Minimum Gas } \\
\text { Flow Rate for Good } \\
\text { Vortex (SLPM) }\end{array}$ \\
\hline \multirow[t]{4}{*}{$0.003175 \times 0.000762$} & 12 & 0.01270 & 0.02540 & 2.00 & $\sim 2.8(\mu-g)$ \\
\hline & & & & & $\sim 2.25$ (lunar-g) \\
\hline & & & & & $\sim 5(1-\mathrm{g})$ \\
\hline & & & & & $\sim 6.5(2-\mathrm{g})$ \\
\hline
\end{tabular}

The results in Table 4.2 show a general trend of increasing flow rate for increasing gravity. This is intuitive since there is more body force in the liquid to overcome in order to create a stable vortex with increasing gravity. It is also more difficult for the vortex to be transferred into the separation chamber in higher gravity. The exception to this trend is the flow rate for microgravity. Fig. 4.1 shows all of the results from the flight experiment. Gravitational acceleration is on the ordinate with units of 1 -g equal to $9.8 \mathrm{~m} / \mathrm{s}^{2}$ and inlet gas flow rate on the abscissa in SLPM. The hollow circles represent a stable vortex, the hollow triangles a parabolic vortex, and the X's a non-stable vortex. It includes microgravity, lunar-gravity, 1-g, and 2-g data. The figure shows lines indicating the transition from Unstable to Parabolic, and from Parabolic to Stable. The lines are approximate but show the basic operating regimes of the separator in different gravitational fields. The value for the minimum flow rate in Table 4.2 for the microgravity test represents the first 
triangle from Fig. 4.1 $1^{5)}$. The transition from Unstable to Parabolic for microgravity is expected to be lower, around 1-2 SLPM, based on the trend line in Fig. 4.1, but the flight experiment did not include a flow rate that low for microgravity.

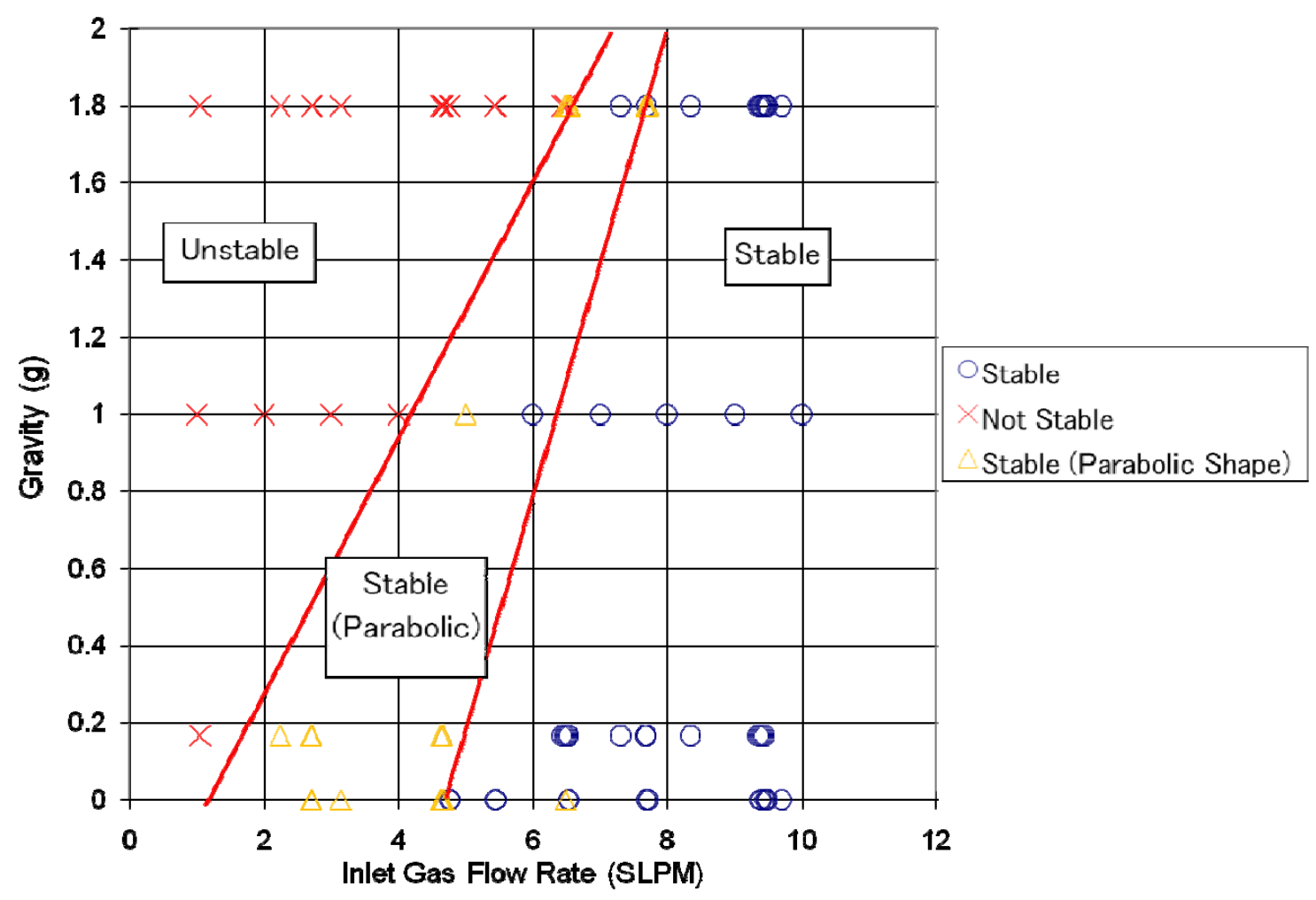

Fig. 4.1 Plot of flight data

Fig. 4.2 shows a plot of the minimum flow rate on the ordinate and the ratio of the separation chamber diameter to the vortex generator diameter on the abscissa. The hollow diamonds represent separators with the $0.00030 \mathrm{~m}$ orifice, the hollow squares represent the $0.00025 \mathrm{~m}$ orifice separators, and the hollow triangle represents the flight tested separator. The error bars presented are described in Appendix B and is the result of the measurement error of the flow meter used and the coarseness of the flow rate increment used in the experiments. The data point at a ratio of 1.71 
and 2 SLPM is shared by both orifice separators. A slight trend of increasing flow rate with increasing diameter ratio appears. This is shown better with the stability line overlaid on the data. This line is determined from the data in Fig. 4.3 which is the results from the dimensional scaling model corresponding to the separator geometries tested. This line marks where the transition is predicted to be between a stable and non stable vortex. If an experimental point is below this line, it indicates that the scaling model over predicted its flow rate, and the converse is true if the point is above the line. The trend of increasing inlet flow with increasing diameter ratio would make sense because as separation chamber size increases relative to the vortex generator size, a larger volume of water would need to be spun. This would require more inlet flow to produce a stronger vortex.



Fig. 4.2 Plot of minimum inlet flow rate for different separator geometries 
Multiple data points for the same diameter ratio exist due to the amount of surface area available for momentum transfer between the vortex and liquid. Assuming that the vortex surface area is that of a cylinder, it scales linearly to the diameter, ignoring the ends. A smaller vortex would have less momentum transfer area and would need to have an increased velocity to compensate for that.

Table 4.1 shows this affect for the diameter ratio of 2.00 for both orifice sizes and the flight tested separator. Some diameter ratios have identical flow rates. This is most likely caused by the inlet flow rate increment used for the experiment being 1 SLPM, resulting in a lack of fidelity to resolve more accurate flow rates.

\section{CFD Results}

Unfortunately, due to licensing issues with Star-CD, CFD results were not able to be obtained. All of the separators were built in Star-CD, and some had completed runs, but the results were unable to be viewed because it required Star-CD viewers. As part of future work, the testing for these separators could be completed and compared to the data presented here.

\section{Analytical Results}

The equations listed in the Theory section (Eq.(2.4) - Eq.(2.10)) were used to calculate the inlet flow rate for each separator geometry.

Table 4.3 lists these results. The flow rates were in the range of 3.10 to 4.39 SLPM. The highest predicted flow rate corresponded to separator 12, the flight separator, and the lowest to separator 7 . Separator 12 had the highest flow rate since it had the largest dimensions, and 7 the lowest since it had the smallest dimensions. 
Table 4.3 Analytical results

\begin{tabular}{|c|c|c|c|c|c|}
\hline Orifice Diameter (m) & $\begin{array}{l}\text { Separator } \\
\text { Number }\end{array}$ & $\begin{array}{c}\text { Vortex Generator } \\
\text { Diameter (m) }\end{array}$ & $\begin{array}{c}\text { Separation } \\
\text { Chamber } \\
\text { Diameter (m) }\end{array}$ & $\begin{array}{c}\text { Separation } \\
\text { Chamber to } \\
\text { Vortex } \\
\text { Generator } \\
\text { Diameter } \\
\text { Ratio }\end{array}$ & $\begin{array}{l}\text { Minimum Gas } \\
\text { Flow Rate for } \\
\text { Good Vortex } \\
\text { (SLPM) }\end{array}$ \\
\hline \multirow[t]{7}{*}{0.00030} & 1 & 0.01111 & 0.02540 & 2.29 & 4.28 \\
\hline & 2 & 0.01111 & 0.02223 & 2.00 & 3.93 \\
\hline & 3 & 0.01111 & 0.01905 & 1.71 & 3.59 \\
\hline & 4 & 0.01270 & 0.02223 & 1.75 & 4.06 \\
\hline & 5 & 0.01270 & 0.01905 & 1.50 & 3.74 \\
\hline & 6 & 0.00953 & 0.01905 & 2.00 & 3.47 \\
\hline & 7 & 0.00953 & 0.01588 & 1.67 & 3.10 \\
\hline \multirow[t]{4}{*}{0.00025} & 8 & 0.01111 & 0.02540 & 2.29 & 4.19 \\
\hline & 9 & 0.01111 & 0.02223 & 2.00 & 3.83 \\
\hline & 10 & 0.01111 & 0.01905 & 1.71 & 3.47 \\
\hline & 11 & 0.00953 & 0.01905 & 2.00 & 3.47 \\
\hline $0.003175 \times 0.000762$ & 12 & 0.01270 & 0.02540 & 2.00 & 4.39 \\
\hline
\end{tabular}




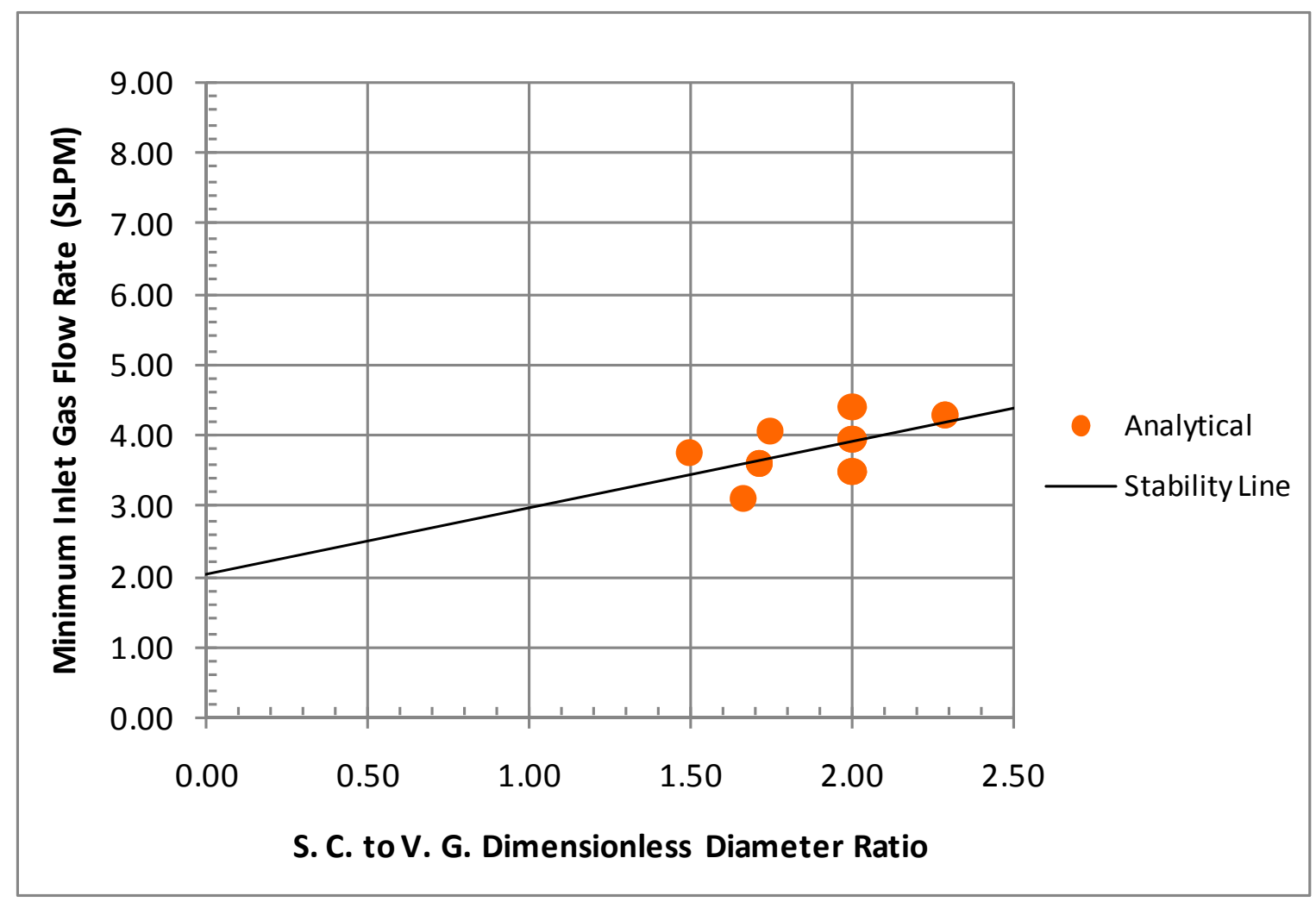

Fig. 4.3 Analytical results of diameter ratio and inlet gas flow rate

Fig. 4.3 shows data similar to Fig. 4.2 with the analytical data in place of the experimental data. The inlet gas flow rate in SLPM is on the ordinate and the diameter ratio on the abscissa. Again, this plot shows that as the diameter ratio increases, so does the inlet gas flow rate. The stability line is based on this data and supports this trend. The stability line is the delineation between a stable and non-stable vortex. This data does not show identical flow rates at different diameter ratios as in Fig. 4.2. 


\section{Data Comparisons}

Table 4.4 Comparison table of the three data sets

\begin{tabular}{|c|c|c|c|c|c|c|c|}
\hline $\begin{array}{l}\text { Orifice } \\
\text { Diameter } \\
\text { (m) }\end{array}$ & \begin{tabular}{|l|} 
Separator \\
Number
\end{tabular} & $\begin{array}{c}\text { Vortex } \\
\text { Generator } \\
\text { Diameter } \\
\text { (m) }\end{array}$ & $\begin{array}{c}\text { Separation } \\
\text { Chamber } \\
\text { Diameter } \\
\text { (m) }\end{array}$ & $\begin{array}{c}\text { Separation } \\
\text { Chamber to } \\
\text { Vortex } \\
\text { Generator } \\
\text { Diameter } \\
\text { Ratio }\end{array}$ & $\begin{array}{l}\text { Experimental Min. } \\
\text { Gas Flow Rate for } \\
\text { Good Vortex } \\
\text { (SLPM) }\end{array}$ & \begin{tabular}{|} 
Analytical Min. \\
Gas Flow Rate \\
(SLPM)
\end{tabular} & $\begin{array}{l}\text { Absolute } \\
\text { Analytical \% } \\
\text { Difference }\end{array}$ \\
\hline \multirow[t]{7}{*}{0.00030} & 1 & 0.01111 & 0.02540 & 2.29 & 5 & 4.28 & $14.42 \%$ \\
\hline & 2 & 0.01111 & 0.02223 & 2.00 & 3 & 3.93 & $-31.14 \%$ \\
\hline & 3 & 0.01111 & 0.01905 & 1.71 & 2 & 3.59 & $-79.41 \%$ \\
\hline & 4 & 0.01270 & 0.02223 & 1.75 & 3 & 4.06 & $-35.37 \%$ \\
\hline & 5 & 0.01270 & 0.01905 & 1.50 & 3.5 & 3.74 & $-6.72 \%$ \\
\hline & 6 & 0.00953 & 0.01905 & 2.00 & 3.5 & 3.47 & $0.84 \%$ \\
\hline & 7 & 0.00953 & 0.01588 & 1.67 & 3 & 3.10 & $-3.48 \%$ \\
\hline \multirow[t]{4}{*}{0.00025} & 8 & 0.01111 & 0.02540 & 2.29 & 4 & 4.28 & $-6.98 \%$ \\
\hline & 9 & 0.01111 & 0.02223 & 2.00 & 4 & 3.93 & $1.64 \%$ \\
\hline & 10 & 0.01111 & 0.01905 & 1.71 & 2 & 3.59 & $-79.41 \%$ \\
\hline & 11 & 0.00953 & 0.01905 & 2.00 & 8 & 3.47 & $56.62 \%$ \\
\hline $\begin{array}{c}0.003175 \\
x \\
0.000762\end{array}$ & 12 & 0.01270 & 0.02540 & 2.00 & 5 & 4.39 & $12.20 \%$ \\
\hline
\end{tabular}

The experimental and analytical results are compared here. Table 4.4 lists the results of each data set and gives a percent difference relative to the experimental measurements. 
For the 1-g case, the data point at 5 SLPM was chosen as the point for comparison in Table 4.4 since it indicated a parabolic shape which is the minimum desired shape for a stable vortex. The Unstable/Parabolic trend line indicates that this flow rate might be smaller, but since there was not an actual measurement there, the point at 5 SLPM was taken.

\section{Analytical to Experimental Comparison}

The largest absolute error encountered was for separators number 3 and 10 at $79.41 \%$. This corresponded to a diameter ratio of 1.71 . The same size of vortex generator and separation chamber was used in both separators, and they both had the same result despite the different orifice size. Although the percent difference in flow rate was the largest, there was only a 1.59 SLPM difference between the analytical flow rate and the measured flow rate. It is believed that finer increments in flow rate during the experimental test would have yielded results that better matched the analytical model. The fact this large error is identical for separators 3 and 10, with the only difference being orifice size, suggests that this combination of separation chamber and vortex generator might not be optimum for vortex coupling and that orifice size is not as important. The second largest absolute error was $56.62 \%$ for separator 11 , which had the largest experimental flow rate at 8 SLPM. The diameter ratio for this separator was 2.00 . Unlike separators 3 and 10 , this flow rate difference was 4.53 SLPM. This measurement was addressed above and is considered invalid for this comparison. The best agreement came for separator 6 at $0.84 \%$. This is the same separator geometry as separator 11 which provided the $56.62 \%$ error, further suggesting that the 8 SLPM measurement was invalid.

Intuitively, the minimum gas flow rate needed for a stable vortex should decrease with S.C. to V.G. ratio. This is what the analytical model predicts, and it makes sense because the vortex can couple better with the separation chamber volume. A larger ratio would cause the vortex to expand to a 
wider diameter and lose more of its rotational momentum thus causing a break down in the vortex. The fact that the ratio of 1.71 in Table 4.4 showed a lower flow rate than the smaller ratios of 1.67 and 1.50 might be caused by vortex flow reversal. In each separator geometry, the separation chamber height was constant, $0.02540 \mathrm{~m}$, but the vortex generator height was equal to its diameter. Therefore, the axial distance available for vortex travel is smaller in the separators with ratios of 1.67 and 1.50. This can lead to increased vortex flow reversal and a corresponding loss of vortex momentum. These results might indicate that the overall height of the separator is crucial to separator performance and should be studied further. 
Table 4.5 Comparison of errors for each separation chamber and vortex generator dimension

\begin{tabular}{|c|c|c|c|c|}
\hline $\begin{array}{c}\text { Orifice } \\
\text { Diameter } \\
\text { (m) }\end{array}$ & $\begin{array}{c}\text { Separation Chamber } \\
\text { Diameter (m) }\end{array}$ & $\begin{array}{c}\text { Average } \\
\text { Absolute Error } \\
\text { (\%) }\end{array}$ & $\begin{array}{c}\text { Vortex Generator } \\
\text { Diameter (m) }\end{array}$ & $\begin{array}{c}\text { Average Absolute Error } \\
\text { (\%) }\end{array}$ \\
\hline \multirow[t]{4}{*}{0.00030} & .02540 & 14.42 & .01111 & 41.66 \\
\hline & .02223 & 33.26 & .01270 & 21.05 \\
\hline & .01905 & 28.99 & .009525 & 2.16 \\
\hline & .01588 & 3.48 & & \\
\hline \multirow{3}{*}{.00025} & .0254 & 4.81 & .01111 & 27.50 \\
\hline & .02223 & 4.15 & .009525 & 56.62 \\
\hline & .01905 & 65.08 & & \\
\hline \multicolumn{5}{|l|}{0.003175} \\
\hline $\mathrm{x}$ & 0.0254 & 12.20 & .01270 & 12.20 \\
\hline 0.000762 & & & & \\
\hline
\end{tabular}

Table 4.5 shows the absolute errors for the different sizes of separation chamber and vortex generator for each orifice size. The flight data in Table 4.5 shows the same error on both sides of the table since only one geometry was tested. The separation chamber diameters listed above are when the vortex generator diameter was constant. For example, the average absolute error for the $0.01905 \mathrm{~m}$ separation chamber and $0.00030 \mathrm{~m}$ orifice was the average for separators with those dimensions and different vortex generator dimensions. The converse is true for the vortex generator sizes. The separation chamber error is largest with a diameter of $0.01905 \mathrm{~m}$ and a 
$0.00025 \mathrm{~m}$ orifice. The vortex generator error is largest at a size of $0.009525 \mathrm{~m}$ and an orifice of $0.00025 \mathrm{~m}$. Again, this is influenced by the large error from separator 11. The diameters are listed in descending order, but the data do not suggest any trend in error with respect to the separation chamber or vortex generator diameters.

Table 4.5 also shows that the error is not significantly more sensitive to separation chamber size changes compared to vortex generator size changes.

Fig. 4.4 and Fig. 4.5 show a comparison of data from Fig. 4.2 and Fig. 4.3. Fig. 4.4 is a comparison of all separators with the $0.00030 \mathrm{~m}$ orifice and Fig. 4.5 of the $0.00025 \mathrm{~m}$ orifice. These figures represent the same flow rate information from Table 4.4 and help to illustrate the difference in the data sets.

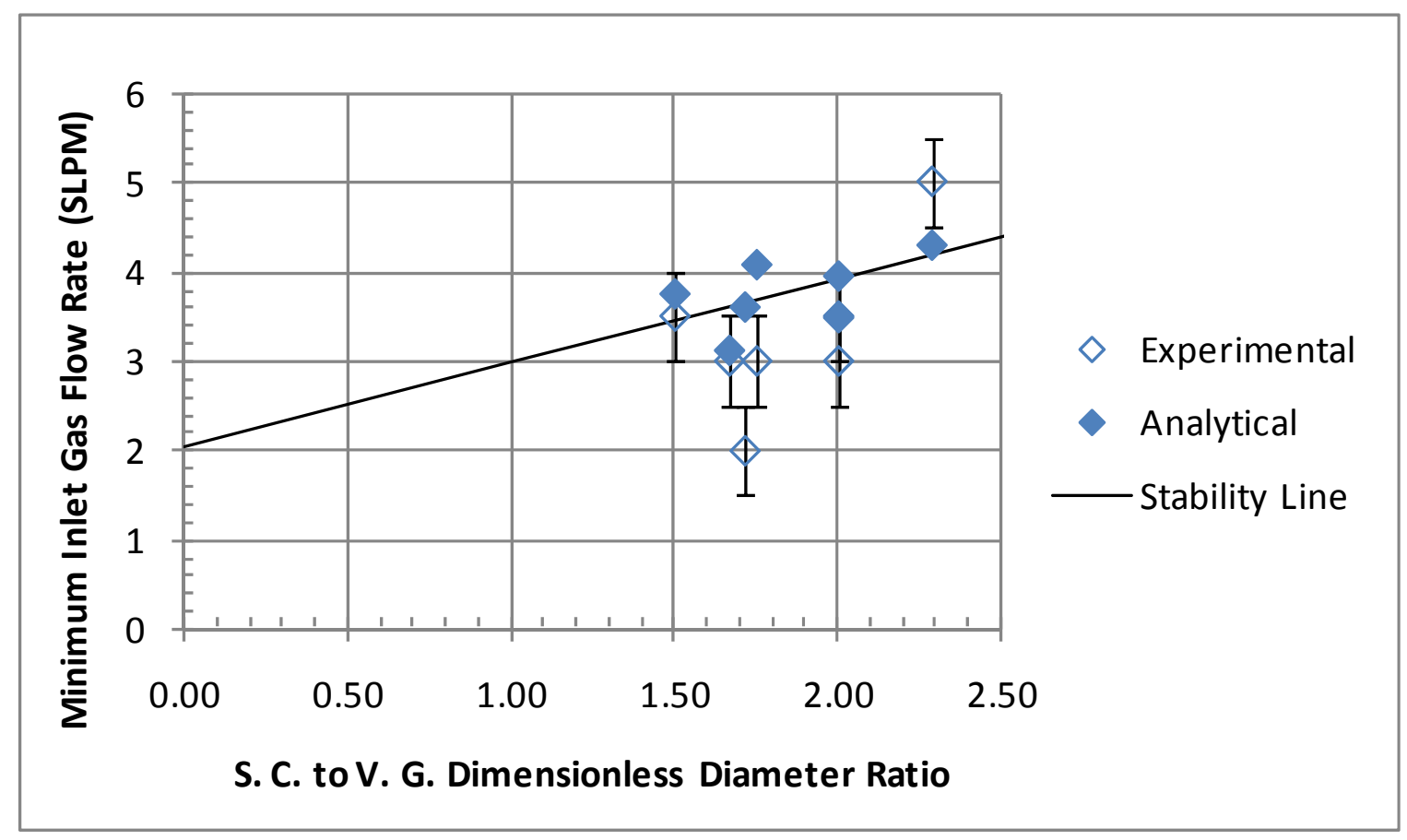

Fig. 4.4 Comparison of experimental and analytical data for the $0.00030 \mathrm{~m}$ orifice 


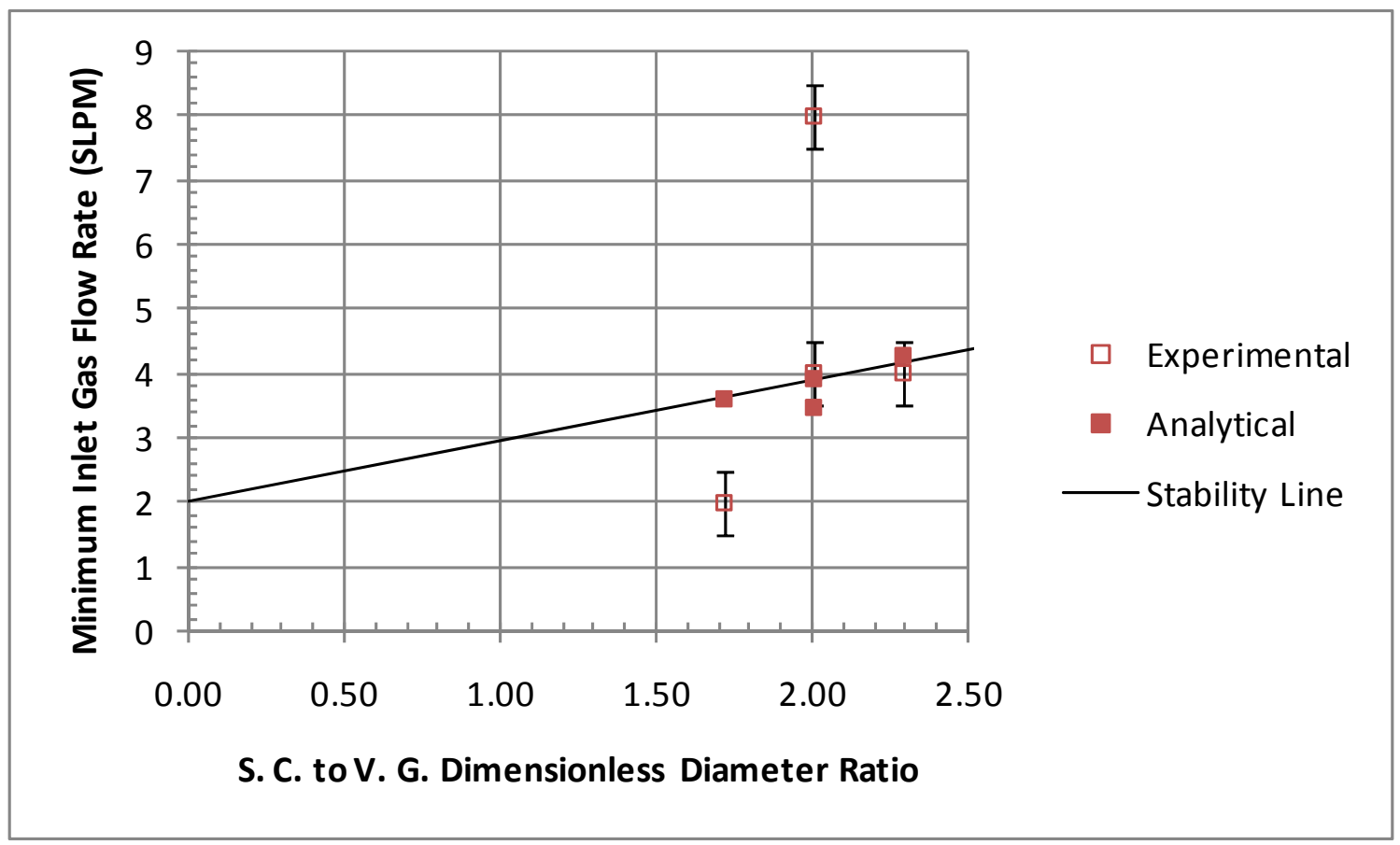

Fig. 4.5 Comparison of experimental and analytical data for the $0.00025 \mathrm{~m}$ orifice

The data for separators with the $0.00030 \mathrm{~m}$ orifice show that the analytical data compares favorably to the experimental data having error of less than $36 \%$ on all but one geometry. This suggests that the analytical model is adequate for these geometries. The separators with the $0.00025 \mathrm{~m}$ orifice compared less favorably, with two of the four geometries having greater than $50 \%$ error. These separators need more data points to determine if the analytical model is sufficient in describing the behavior of these separators. Also, refinement of the measurements of inlet flow rate would give better results for comparison.

The main energy loss mechanism of the vortex is through shear stress between the vortex generator wall and the gas-liquid interface in the separation chamber. Other losses include vortex expansion as it travels from vortex generator to the separation chamber and vortex flow reversal as it hits the 
top of the separation chamber. Table 4.4 shows that the analytical model predicts higher flow rates for separators with identical separation chambers and larger vortex generators. This makes sense because a larger vortex generator correlates to greater shear losses on the wall. Also, separators with identical vortex generators and larger separation chambers require larger inlet flow rates. This is due to two main reasons: a greater mass of liquid to rotate, and a larger surface area for interfacial shear causing greater shear losses.

One method of estimating the interfacial shear stress could be by assuming the flow can be modeled as concurrent annular flow. Wallis ${ }^{6}$ gives an equation for calculating shear stress in this situation.

$$
\tau_{i}=\frac{\left(C_{f}\right)_{i} \rho_{g} v_{g}^{2}}{2}
$$

where $\tau_{i}$ is the interfacial shear stress, $\left(C_{f}\right)_{i}$ is the interfacial friction factor, and $v_{g}$ is the gas velocity at the interface. The equation for $\left(C_{f}\right)_{i}$ is

$$
\left(C_{f}\right)_{i}=\alpha^{5 / 2} \phi_{g}^{2}\left(C_{f}\right)_{g}
$$

where $\alpha$ is the void fraction, $\phi_{g}$ is the gas Martinelli Parameter, and $\left(C_{f}\right)_{g}$ is the friction factor if the flow was all gas, i.e. the interface becomes a wall. 
Equation (2.6) shows the minimum angular velocity needed for a stable vortex which could be used to calculate the shear stress in this situation. Then, using Eq. (2.10) this shear stress could be related to the inlet gas flow rate. In this way, the coupling between the vortex generator and separation chamber could be better understood. 


\section{CONCLUSION}

Phase separation is an important process in a variety of different applications both terrestrial and in outer space. Many apparatuses have been invented to perform this function, each with their own advantages and disadvantages. Earthly uses of separators include BWR power plants, the petroleum industry, and the cement industry, to name a few. All of these separators employ gravity to aid in separation.

The design of Fig. 1.1 and Fig. 1.2 from the Introduction section has experienced thousands of parabolas of rigorous testing aboard NASA's KC-135 and DC-9 reduced gravity aircraft. It performs well when liquid is the driving flow and can be designed to accommodate myriad of system conditions. It suffers performance degradation when liquid flow is at a minimum, but gas flow is high. Such an application could exist in a system involving a proton exchange membrane fuel cell (PEMFC) when exhaust/sweeping flow of oxygen needs to be separated from exhaust water.

The Interphase Transport Phenomenon (ITP) laboratory at Texas A\&M University (TAMU) invented a vortex separator to be applied in microgravity environments, where gas flow is the driving flow of the separator, a new design was created, (Figure 1.3 from the Introduction section). This thesis performed a study to learn more details on the operating range of this separator. To do this, experiments were conducted on the ground and in a microgravity environment to determine the minimum inlet gas flow rate necessary to produce a vortex capable of phase separation. An analytical model was formulated to predict the minimum flow rate, and was compared to the experimental data. The separator was then built using the CFD package STAR-CD, but computational runs were not able to be completed due to licensing issues of the software. 
The experiments conducted tested eleven different separator geometries, seven using a $0.00030 \mathrm{~m}$ orifice and four using a $0.00025 \mathrm{~m}$ orifice. A separator was tested aboard NASA's microgravity aircraft. All of the separators had two main components: the vortex generator and separation chamber. The vortex generators had diameters of, $0.01270 \mathrm{~m}, 0.01111 \mathrm{~m}$ and $0.00953 \mathrm{~m}$, while the separation chambers had diameters of $0.02540 \mathrm{~m}, 0.02223 \mathrm{~m}, 0.01905 \mathrm{~m}, 0.01588 \mathrm{~m}$, and 0.01270 m. For each particular test geometry, the separator was filled to a level approximately $1 / 4$ the height of the separation chamber, and the inlet gas flow rate was increased in a step-wise fashion from 110 SLPM and then back down from 10-1 SLPM. At each flow rate, the vortex was observed and rated for acceptability.

The separator designs were modeled using a computer aided design (CAD) program and imported into STAR-CD. A model was built to match each of the twelve geometries tested experimentally. Each one would have been subjected to the same testing procedure as described in the experiment if not for the licensing problems.

The analytical model was developed based on the inlet flow being converted into a vortex, and using the Weber number to determine the minimum rotational speed required for an acceptable vortex. Based on this method, a minimum flow rate was predicted for each separator and compared to the experimental results. Of the twelve separators, nine had errors less than $36 \%$, and the lowest was $0.84 \%$. This implies that the analytical model is reasonable for these geometries. Three separators had errors of greater than $50 \%$ suggesting that the model might not be adequate for these geometries.

The purpose of this thesis was to develop a better understanding of the new separator design and create a methodology/model to predict its performance under different inlet flow conditions. The experiments provided baseline data to compare with analytical models. The analytical model 
proved to be an accurate representation of the separator in most cases. Therefore, these models can provide a basis for future design work on separators.

The analytical results suggest that the closer in diameter the vortex generator and separation chamber are, the lower the minimum flow rate necessary for an acceptable vortex. From this, it is recommended that whenever possible, the separation chamber to vortex generator diameter ratio be as close to unity as possible. Reaching unity will cause the design to go back to that shown in Fig. 1.1 which us undesired. The experimental data loosely follows this recommendation.

This study was only a first look into the behavior of this separation device. Many more attributes could be examined. The separators here had separation chambers that were not right cylinders. The previous separator design of ITP does have this feature. Including this on the new design could affect the strength of the vortex created and the overall liquid capacity that the separator could handle. A more detailed experiment that measured inlet flow rate in finer increments would also provide for better comparison with the analytical model. Completing the CFD runs would add another dimension to this study and provide another tool to design the separator to fit a flow loop's needs. 


\section{REFERENCES}

1. J. McQuillen, "Gas-liquid Flows and Phase Separation," Strategic Research to Enable NASA's Exploration Missions Conference and Workshop: Presentations, 1, 587-611 (2004).

2. W. Dean, "Zero Gravity Phase Separator Technologies--Past, Present and Future," SAE Technical Paper Series, 921160, 1-7 (1992).

3. M. Ellis, "The Tangential Velocity Profile and Momentum Transfer Within a Microgravity Vortex Separator," M.S. Thesis. Texas A\&M University, (2006).

4. D. Bean, F. Best, C. Kurwitz, P. Waghela, "Vortex Necking Phenomena Under Microgravity," Proceedings of the Thermal Fluids Analysis Workshop 2002, NASA Johnson Space Center, (2002).

5. F. R. Best, R. Oinuma, C. Kurwitz, "Final Report of Development of Water Removal System from Oxygen Recirculation Loop Polymer Electrolyte Membrane Fuel Cell," College Station, Texas A\&M University (2008).

6. G. B. Wallis. One-dimensional Two-phase Flow, McGraw-Hill, New York (1969).

7. M. Reyes-Gutierrez, L. Rojas-Solorzano, J. Marin-Moreno, A. Melendez-Ramirez, J. Colmenares. "Eulerian-Eulerian Modeling of Disperse Two-phase Flow in a Gas-liquid Cylindrical Cyclone," Journal of Fluids Engineering, 128, 832-837 (2005).

8. H. F. Meier, A. A. Vegini, J. J. Iess, M. Mori, "Computational Fluid Dynamics (CFD) Analysis of Cyclone Separators Connected in Series," Industrial and Engineering Chemistry Research, 47, 192-200 (2008). 
9. G. Massarani, Fluidodinamica em Sistemas Particulados, Editora UFRJ, Rio de Janeiro (1997).

10. H. F. Meier, "Modelagem Fenomenologica e Simulacao Bidimensional de Ciclones por Tecnicas da Fluidodinamica Computacional," Ph.D. Dissertation. Universidad Estadual de Campinas, Brazil, (1998).

11. Methodology: Star-CD Version 4.02, CD-adapco, London (2006).

12. Meshing User Guide: Star-CD Version 4.02, CD-adapco, London (2006). 


\section{APPENDIX A}

\section{USE OF COMPUTATIONAL FLUID DYNAMICS}

A previous thesis by Ellis ${ }^{3)}$ used STAR-CD to analyze the single chamber separator design, specifically examining the effects of varying inlet flow rate and separator geometry on the tangential velocity profile. He ran cases involving three different separator diameters $(14 \mathrm{~cm}, 11.4$ $\mathrm{cm}$, and $5.1 \mathrm{~cm})$, five different nozzle geometries $(0.457 \mathrm{~mm}$ x $4.57 \mathrm{~mm}, 1.02 \mathrm{~mm} \times 10.2 \mathrm{~mm}$, $0.330 \mathrm{~mm} \times 10.2 \mathrm{~mm}, 1.24 \mathrm{~mm} \times 12.4 \mathrm{~mm}$, and $0.404 \mathrm{~mm} \times 12.4 \mathrm{~mm}$ ) and varying inlet volumetric flow rates (0.58 LPM-10 LPM). Ellis took the cell-centered tangential velocities and averaged them over the separation volume to produce an average rotational speed for each calculation run and compared these to experimentally measured rotational speeds. In general, the CFD calculated rotational speeds followed the same shape as the measured values, but for the two smaller separator diameters, the CFD results over predicted the rotational speed. This is most likely due to the intrusive measurement system used to measure the rotational speed in the experiments. It introduced an additional momentum loss mechanism which manifested itself greater in the smaller diameters. Ellis' application of CFD is important because it involves a gasliquid vortex separator similar to the one being studied in this thesis and it uses the same software package.

The petroleum industry also employs gas-liquid cylindrical separators and study of these separators is ongoing. Reyes-Gutierrez et al. ${ }^{7)}$ utilized the CFX4.3 software package from Ansys to analyze the free surface and tangential velocity inside a typical gas-liquid cylindrical cyclone separator as patented by the University of Tulsa in 1994. Both a single phase and two-phase simulation were used to compute the azimuthal velocity profile. In the single phase simulation, comparison of the turbulence k- $\varepsilon$ model and the low Reynolds k- $\varepsilon$ model showed little difference between the two. 
The single phase CFX results compared with experimental results showed a maximum relative error of $27 \%$ and an average relative error of $13 \%$. The two-phase model showed better agreement between the mean angular velocities from CFX and experimental results with a maximum relative error of $17.6 \%$ and an average of $11 \%$. Examination of the free surfaces produced by CFX and the experiments showed good agreement. A note that Reyes-Gutierrez et al. had was that the shapes of the vortices in their work were helical cones as opposed to axi-symmetric paraboloids or cylinders as is commonly assumed in flow modeling. This difference might have contributed to the errors they experienced.

Another industry that takes advantage of vortex separators is the cement industry. When manufacturing cement, the raw material must be heated, and vortex separators are used for this. There are two main differences with these separators and those mentioned thus far: these separators operate in terrestrial g-fields, and these devices separate gas and solid. The solid is raw meal particulate and the gas is air heated to several hundred degrees. Meier et al. used $\mathrm{CFD}^{8)}$ for separators connected in series to calculate separation efficiency and pressure drop for each separator. They verified their CFD results with data from industry. For their CFD model, there are four key assumptions: the solid particles are represented by a mean diameter, the turbulence of the gas phase is anisotropic, a combination of the k- $\varepsilon$ standard model and Prandtl's longitudinal mixing model can be used, and that perturbations in the flow near the inlet region quickly disappear which allows the use of axial symmetry and the 3-D symmetry model. For the shear stress calculations, a Boussinesq approximation was used where the stress tensor is directly proportional to the deformation rate and the viscosity was a combination of the molecular and turbulent viscosities. The drag force between the solid particles and the gas was calculated with a correlation by Coelho and Massarani ${ }^{9)}$ because it is applicable for all flow regimes. The heat transfer coefficient was calculated using a correlation from Peres and Cremasco. The boundary conditions considered in 
the analysis were a uniform inlet profile, no-slip conditions on the wall, axial symmetry, and continuity conditions at the exits. Single phase flow was first simulated in order to get initial conditions for two-phase flow. The initial conditions for the single phase flow were that velocity was equal to zero and the pressure field was atmospheric. The steady state flow profile of this single phase flow was used as the initial condition of the two-phase flow with an abrupt infusion of solid particles. The software to perform the CFD calculations was CYCLO $^{10)}$ which was developed by Meier, and it uses a finite volume approach. The Semi-Implicit Method for Pressure Linked Equations Consistent (SIMPLEC) algorithm was used to couple pressure and velocity. In general, their results showed good agreement with industry data with deviations less than or equal to $25 \%$ in separation efficiency and pressure drop.

\section{CFD Theory}

\section{Methodology}

To simulate the operation of the separator, the software package pro-STAR developed by CDadapco was used. Pro-STAR is a pre- and post-processor for CFD analysis that offers three different models for multiphase flow: Lagrangian, Eulerian, and the Volume of Fluid (VOF) method. The VOF method was chosen for this application because it solves for the free surface between two immiscible fluids which is one of the main interests in this investigation. The other two methods are geared more towards dispersed multiphase flow. This type of flow does occur during startup of the separator and during transients, but does not occur significantly during normal steady state operation.

Specifically, the VOF method is meant to compute a well-defined free surface between two immiscible fluids such as that found in stratified flow in a pipe, or transitional flows where the free surface is disturbed and the two phases can mix to some extent. ${ }^{11)}$ The assumptions that apply to 
the pro-STAR VOF method are: (1) that there only exist two fluid components in the system, (2) each fluid has constant density, (3) and all components contained in any control volume share the variables for the mixture by solving the transport equations for velocity, pressure, temperature, etc. The second assumption is weakly valid in the nozzle portion of the separator, but is reasonable for the majority of the separator volume. As stated, only two fluids are allowed with the VOF method, but the fluids may both be liquids which are immiscible or gases. In any case, one fluid is labeled the "heavy fluid" and the other the "light fluid." In the separator, the heavy fluid is water and the light fluid is air. If a liquid phase is present and cavitation occurs, then the resulting vapor is added as a third component automatically. This phenomenon does not occur in the separator because the pressures remain above the saturation pressure for water at operating conditions. Other fluid properties besides the density are allowed to vary when necessary, such as viscosity, thermal conductivity, specific heat, etc. For the separator, these values were kept constant and at the standard values at room temperature. The semi-implicit method for pressure linked equations (SIMPLE) algorithm for transient calculations is the only algorithm available to solve the conservation equations when using the VOF method.

Pro-STAR uses the finite volume (FV) method to solve the conservation equations in the discretized problem domain, and uses a coordinate free form of these equations to ensure versatility in its applications. This coordinate free version is given in Eq. (A.1 $)^{\mathbf{1 1})}$

$$
\frac{\partial}{\partial t}(\rho \phi)+\nabla \bullet\left(\rho \vec{u} \phi-\Gamma_{\phi} \nabla \phi\right)=s_{\phi}
$$

where $\vec{u}$ is the fluid velocity vector, $\phi$ is any dependent variable, $\Gamma_{\phi}$ is the associated diffusion coefficient, and $s_{\phi}$ is the associated source coefficient. For an arbitrary time varying volume $V$ which is bounded by a moving closed surface $S$, an exact solution to Eq. (A.1) is 


$$
\frac{d}{d t} \int_{V} \rho \phi d V+\int_{S}\left(\rho \vec{u}_{r} \phi-\Gamma_{\phi} \nabla \phi\right) \bullet d \vec{S}=\int_{V} s_{\phi} d V
$$

where $\vec{S}$ is the surface vector of surface $S$, and $\vec{u}_{r}$ is the relative velocity between the fluid velocity, $\vec{u}$, and the surface $S$ velocity, $\vec{u}_{c}$. This relative velocity is given by Eq. (A.3). For the separator, $\vec{u}_{c}$ is zero.

$$
\vec{u}_{r}=\vec{u}-\vec{u}_{c}
$$

An example of two arbitrary cells in a discretized problem domain is shown in Fig. A.1. For cell $A$, the volume is $V_{A}$ and there are $S_{j}$ discrete faces. With these definitions, Eq. (A.2) transforms into

$$
\underbrace{\frac{d}{d t} \int_{V_{A}} \rho \phi d V}_{T_{1}}+\underbrace{\sum_{j} \int_{S_{j}}\left(\rho \vec{u}_{r} \phi-\Gamma_{\phi} \nabla \phi\right) \bullet d \vec{S}}_{T_{2}}=\underbrace{\int_{V_{A}} s_{\phi} d V}_{T_{3}}
$$

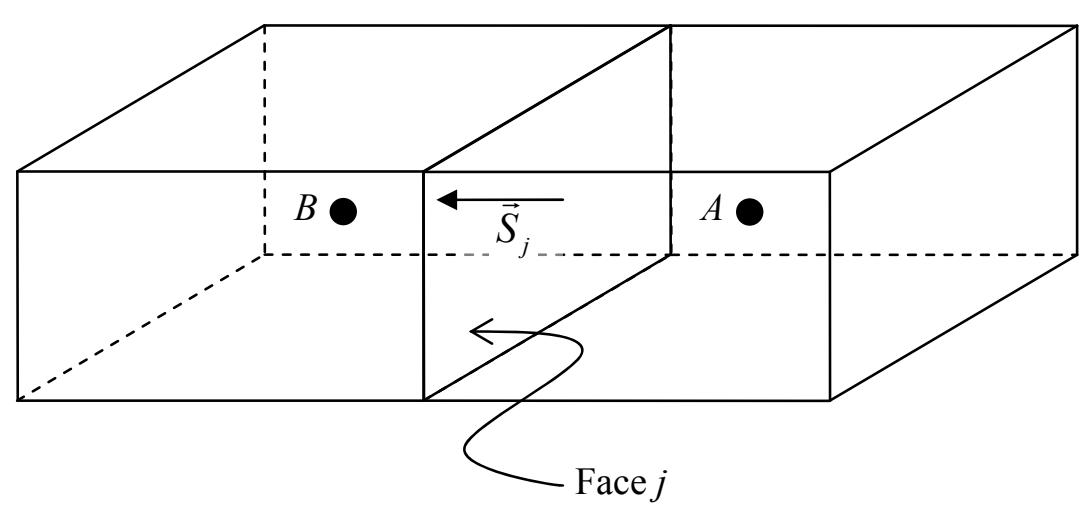

Fig. A.1 Representation of two arbitrary neighboring cells 
For the complicated geometries and flow conditions of the separator, the exact equation from Eq. (A.2) will not be solvable, so approximations are necessary. The term $T_{2}$ in Eq. (A.4) can be classified into the convection and diffusion terms, $C_{j}$ and $D_{j}$ respectively. These terms are approximated averages over the cell faces. Therefore, $T_{2}$ can be approximated as

$$
T_{2} \approx \sum_{j}\left(\rho \vec{u}_{r} \phi \bullet \vec{S}\right)_{j}-\sum_{j}\left(\Gamma_{\phi} \nabla \phi \bullet \vec{S}\right)_{j} \equiv \sum_{j} C_{j}-\sum_{j} D_{j}
$$

This approximation represents the midpoint rule for approximating surface integrals which is accurate to the second order. The diffusion term $D_{j}$ is broken down into normal diffusion and cross diffusion between $A$ and $B$. The first term in Eq. (A.6) is the normal diffusion and the term in curly brackets is the cross diffusion.

$$
D_{j} \approx \Gamma_{\phi, j}\left[f_{j}^{l}\left(\phi_{B}-\phi_{A}\right)+\left\{\nabla \phi \bullet \vec{S}-f_{j}^{l} \nabla \phi \bullet \vec{d}_{A B}\right\}_{j}\right]
$$

In Eq. (A.6), $f_{j}^{l}$ is a geometry factor calculated by pro-STAR, $\vec{d}_{A B}$ is the distance vector from $A$ to $B$, and $\Gamma_{\phi, j}$ is the interpolated diffusion coefficient for each cell face. Explain more about $f_{j}^{l}$.

There are several options for the discretization of the convective and diffusive terms of Eq. (A.5). In problems that involve high Reynolds number flows, the selection of a discretization scheme becomes more important for the convective term. These available schemes for convective flux approximations are broken up into two categories: first order and second order. Low order schemes create equations that are relatively simple to solve, and their solutions obey physical bounds. However, low order schemes can create smearing of gradients, a phenomenon known as numerical diffusion. This effect is a result of the truncation error inherent in low order schemes and can be reduced with finer meshes. High order schemes are useful when steep gradients are 
present, but they produce more complex equations to solve that could become unstable and result in non-physical solutions. Again, a finer mesh can remedy this situation.

The convective term, $C_{j}$, is expressed as

$$
\begin{gathered}
C_{j} \equiv F_{j} \phi_{j} \\
F_{j} \equiv\left(\rho \vec{u}_{r} \bullet \vec{S}\right)_{j}
\end{gathered}
$$

These equations reflect Eq. (A.5). The term $F_{j}$ represents the mass flux through face $j$, and $\phi_{j}$ is the average value of the dependent variable at face $j$. This is computed by interpolation of the appropriate neighboring nodal values based on the differencing scheme used. The values of $\rho$ and $\Gamma$ at face $j$ are also calculated by interpolation.

Issues of having non-real results in previous calculation runs of the separator have led to the selection of the Upwind Differencing (UD) scheme for this application. The UD scheme is a first order scheme, which obeys physical bounds, that takes the nearest upwind neighbor value for $\phi_{j}$ for use in the formulation of the convective flux.

$$
C_{j}^{U D} \equiv F_{j} \begin{cases}\phi_{N}, & F_{j} \geq 0 \\ \phi_{N+1}, & F_{j}<0\end{cases}
$$

The diffusive term, $D_{j}$, and $T_{3}$ term of Eq. (A.4) are treated in a similar way. 


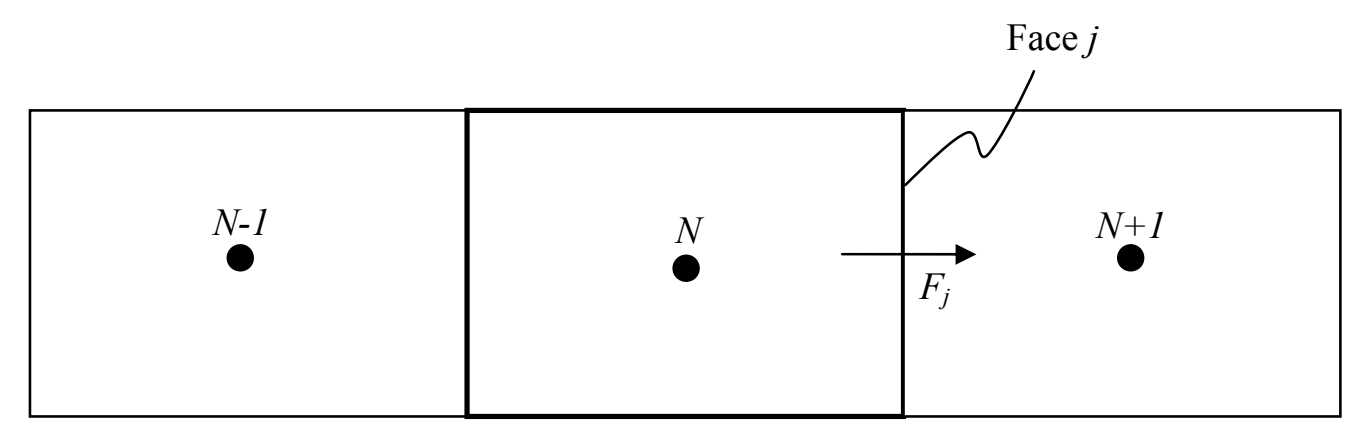

Fig. A.2 Diagram showing the convention for node labeling for Eq. A.9

To approximate the time derivative of the problem, pro-STAR offers three schemes: the fully implicit Euler scheme, the quadratic backward implicit scheme, and a special form of the fully implicit Euler (FIE) scheme that involves explicit deferred correctors. The first two options are only available when using the SIMPLE solver and the third option is only available when using the PISO solver. Since the SIMPLE solver is used for this analysis, the PISO time derivative approximation will not be explained. Additionally, while using the VOF method, only FIE is allowed to be used, so only it will be expounded on.

With FIE, the time derivative is converted into a simple difference relation given in Eq. (A.10). The $T_{l}$ term of Eq. (A.4) is approximated as a liner variation between two different times, $t=i$ and $t=i-1$. This method is a first order approximation, and it eliminates stability related time step restrictions in the calculation. It should be noted that values of $T_{2}$ and $T_{3}$ are calculated at $t=i$.

$$
T_{1}=\frac{(\rho \phi V)^{i}-(\rho \phi V)^{i-1}}{\delta t}
$$


These sets of equations are formulated and solved for each node in the problem domain for each time step. The process begins for each time step at the cells where the inlet faces are defined and terminates at the cells which define the outlet.

To use the VOF method, an initial void fraction must be defined. This can be done either by defining different regions in the problem domain as being the heavy/light fluid, or a void fraction can be defined that will apply to each cell in the calculation. For the simulations of the separator, the former option was chosen. Fig. A.3 shows how the separator was modeled in pro-STAR.

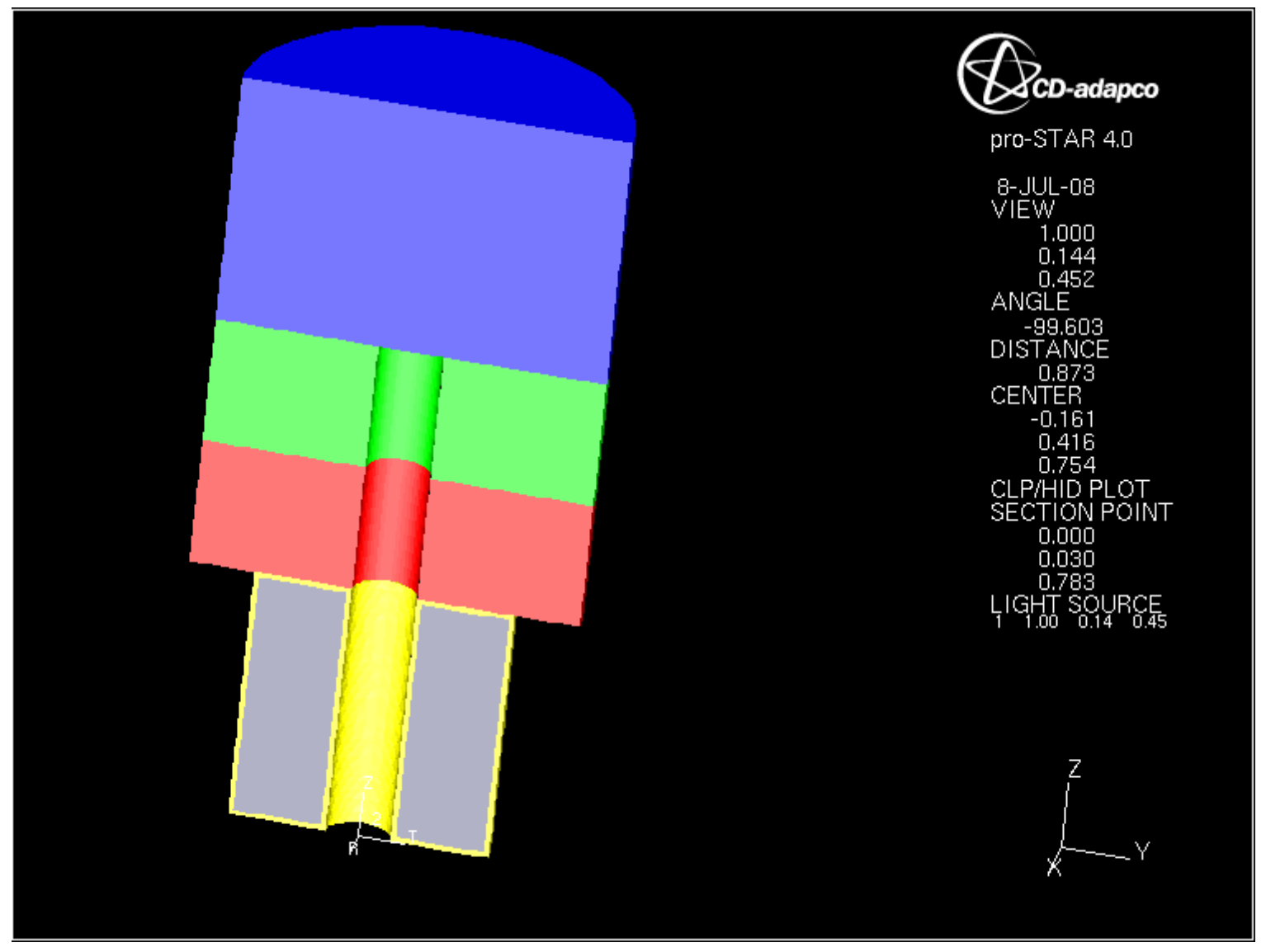

Fig. A.3 Schematic of the separator showing the different regions 
The vortex generator is composed of two different types of cells, the prism layer cells and the core volume cells. Defining the prism layer as water contributed to a divergent solution because of the instability of the inlet gas jet penetrating the cells. For this reason, the prism layer was defined as gas. These comprise a very small fraction of the volume in the vortex generator, so defining these cells as gas is not believed to cause appreciable error. In addition, during the steady state operation of the separator, the vortex generator has very little liquid in it, so these cells would ultimately become gas. The core volume cells in the vortex generator were initially defined as water. The red region in Fig. A.3 was initially defined as liquid, and its height one quarter the total separation chamber height. The blue and green regions were initially defined as gas and cover the remaining height of the separation chamber with the green region being one quarter of the total height and the blue one half. In this way, the initial volume fractions are defined as

$$
\alpha_{i}=\frac{V_{i}}{V}
$$

where $V_{i}$ is the volume of component $i$ in the control volume $V$. The volume fraction of the other component is simply one minus $\alpha_{i}$. For the separator, $\alpha_{l}$ for the red and core volume regions is unity and $\alpha_{g}$ is zero, and the converse is true for the blue and green regions. Using these volume fractions, the effective properties in each cell are calculated. Examples of the effective density and specific heat in a cell are

$$
\begin{gathered}
\rho=\alpha_{g} \rho_{g}+\alpha_{l} \rho_{l} \\
c_{p}=\frac{\alpha_{g} \rho_{g} c_{p, g}+\alpha_{l} \rho_{l} c_{p, l}}{\rho}
\end{gathered}
$$


Viscosity and the other effective properties are calculated similarly. These effective properties are then used in their respective single phase transport equations and vary strongly across the gasliquid interface. In this way, the free surface is determined.

A special algorithm called the High-Resolution Interface Capturing (HRIC) scheme is used for the convective terms, $C_{j}$, of the conservation equations. This scheme calculates the cell-face volume fraction as a weighted average of the upstream and downstream cell-center values. The weighting factor is determined by the gas-liquid interface orientation relative to the cell face, the local Courant number, and the profile of $\alpha_{i}$ near the cell face. The Courant number is a guideline on how to set the time step for a transient analysis. It specifies that the time step must be less than the amount of time it takes for a fluid to travel through a cell. Pro-STAR recommends that the Courant number should remain below approximately 0.3 for accurate results. As the Courant number increases, the interface will smear more and more. Equation (A.14) defines the Courant number.

$$
C o=\frac{u_{x_{i}} \Delta t}{\Delta x_{i}}
$$

where $u_{x_{i}}$ is the velocity of the fluid in direction $x_{i}, \Delta t$ is the time step, and $\Delta x_{i}$ is the change in the arbitrary direction $x_{i}$.

\section{Grid Creation}

The formation of the grid for the separator geometry was split between the vortex generator and the separation chamber. For the vortex generator, the geometry was imported into pro-STAR and the auto-meshing function was used to create the mesh. This approach was chosen because of the complexity of the geometry near the inlet nozzle. The three types of mesh available through the 
auto-mesher are trimmed, polyhedral, and tetrahedral. ${ }^{12)}$ The trimmed mesh is the default option in the auto-mesh module and it offers advantages such as using predominantly hexahedral cells, minimal cell skewness, automatic curvature and proximity refinement, and surface quality independence. Near a surface, the hexahedral cell can be trimmed to better match the surface shape. In general, these meshes create finer cells around edges and surfaces while leaving core regions with coarser cells. This is undesirable for the vortex generator since fidelity is required not only on the periphery, but in the core region as well. In the polyhedral mesh, an initial tetrahedral grid is created on the imported geometry's surface from which the polyhedrons are based. A dualization scheme is used to create the mesh, and the initial tetrahedral surface grid is replaced by the resulting polyhedral mesh. On average, the polyhedrons have 14 faces. Polyhedral meshes help to give balanced solutions when a problem requires complex mesh generation. A tetrahedral mesh is built on the initial tetrahedral surface grid created when the geometry is imported. Tetrahedral meshes offer the fastest meshing times and the least amount of memory required compared to the other two meshing strategies.

Each type of mesh was considered and attempted for the vortex generator. The trimmed mesh lacked resolution towards the center of the vortex generator and was thus eliminated from consideration. There are options available to refine a trimmed mesh to provide detail in any region of a problem domain, but the process for this is not justifiable compared to the simplicity of the processes of the other two meshing strategies. The polyhedral mesh created a very fine and detailed mesh and was the original choice for the vortex generator. However, calculation times for this complex mesh were prohibitively long. The best alternative for the vortex generator mesh was the tetrahedral mesh. It provided sufficient detail throughout the vortex generator, including the inlet nozzle, and an adequate number of cells to allow for reasonable calculation times. The tetrahedral mesh for the $1 / 2{ }^{\prime \prime} \mathrm{Dx}^{1} / 2$ " $\mathrm{H}$ is shown in Fig. A.4. The cluster of cells to the left and center 
of the vortex generator is where the nozzle entrance is located. It is shown in Fig. A.4 that there are two cell types, the prism layer (yellow) and the core cells (grey). The prism layer is an extrusion of the initial tetrahedral mesh from which the core cells are created. A prism layer is required when using the meshing strategies described above, but options exist to modify the prism layer to get more accurate results.



Fig. A.4 A clipped view of the interior tetrahedral grid for the 1/2"Dx1/2"H vortex generator

The separation chamber used a different meshing strategy. The initial water level of the separator was desired to be controlled, so three zones in the separation chamber were created. The bottom- 
most (red) zone was created first and it was one quarter of the total height of the separation chamber. This zone was divided into 20 radial slices, 50 tangential slices, and 15 axial slices. The inner radius of this zone butted against the gas outlet tube. In calculation runs, this zone was set to be all liquid. The middle zone (green) was identical to the previous zone in composition except, during calculations, it was set to be all air. These two zones together comprised half of the axial height of the separation chamber. The remaining volume of the separation chamber (blue) had the same divisions as the previous zones except there were 30 axial slices since this volume had twice the axial height. This zone also did not butt radially against the gas outlet tube, instead, it formed the top of the tube. Fig. A.5 illustrates these zones and their meshing. The total number of cells in the separation chamber remained constant for each separator size, but the number of cells in the vortex generator varied depending on its size.

Fig. A. 7 through Fig. A.9 show the three types of zones in the separation chamber looking down axially on them. The red and green pieces were donut shaped where the hole in the middle represents the boundary of the gas outlet tube. The blue region did not have a hole in the center, and it defined where the top of the gas outlet tube resided. 


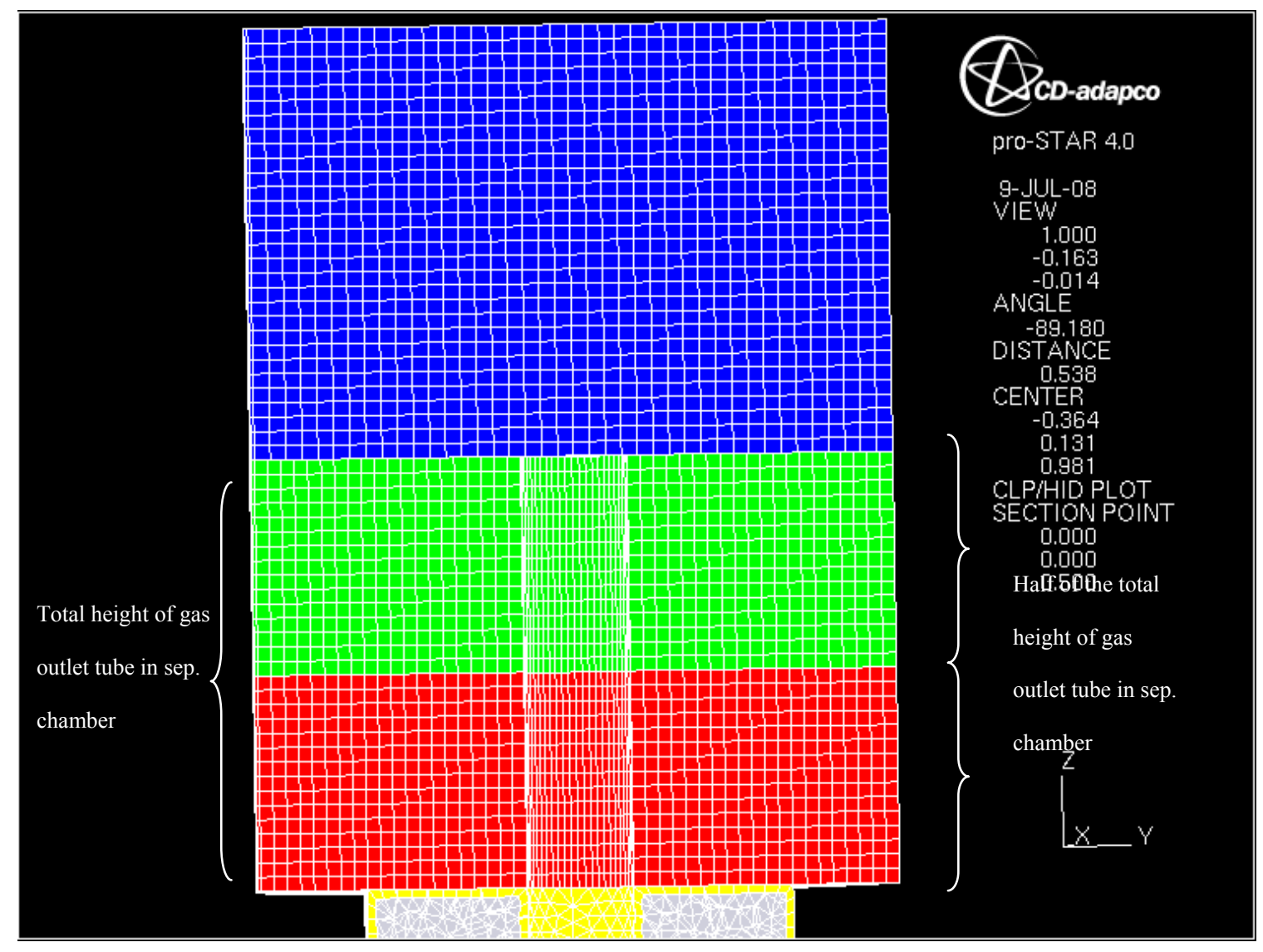

Fig. A.5 A clipped view of the mesh inside of the 3/4"Dx1"H separation chamber 


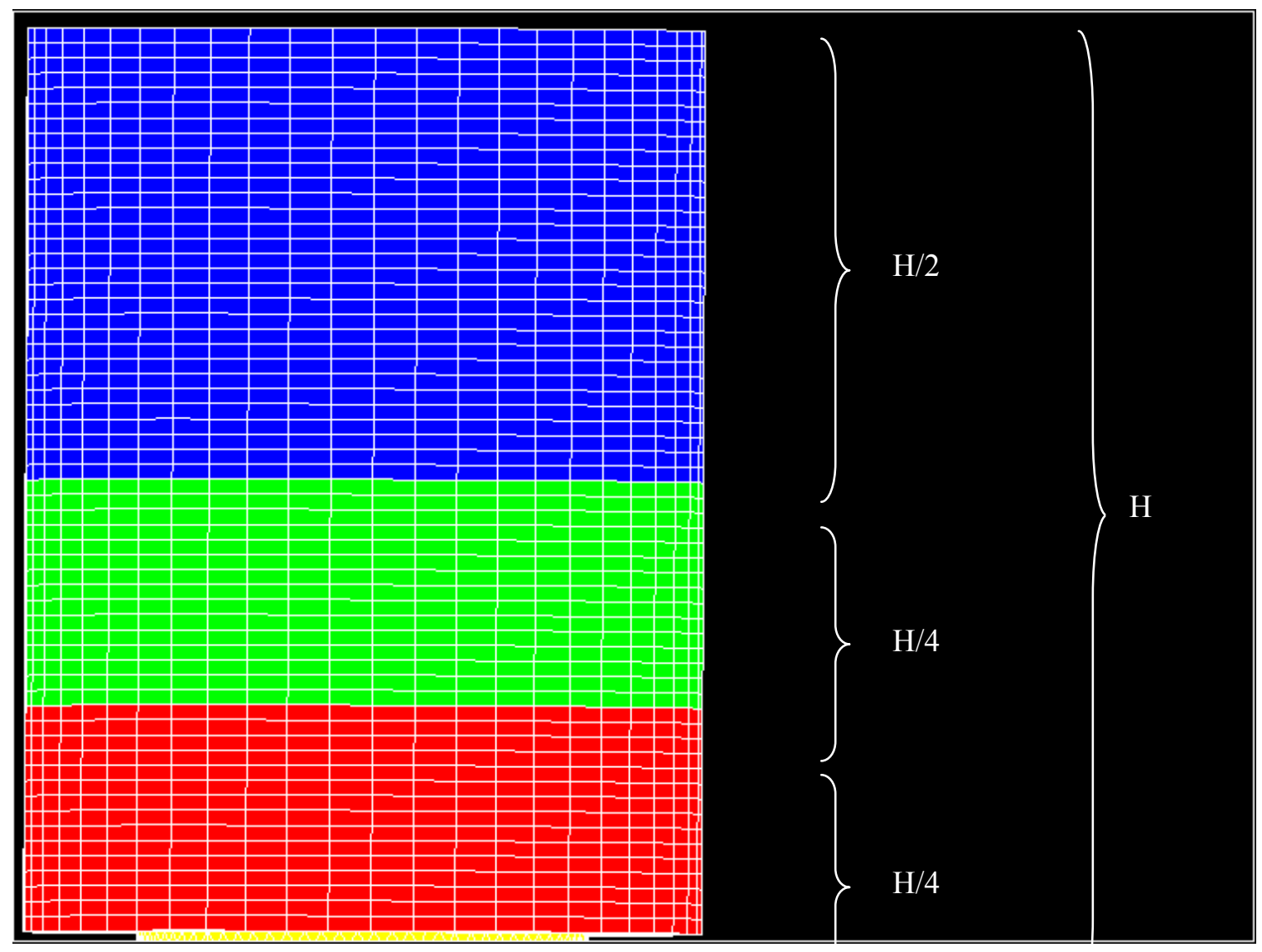

Fig. A.6 Separation chamber and the relative dimensions of each zone 


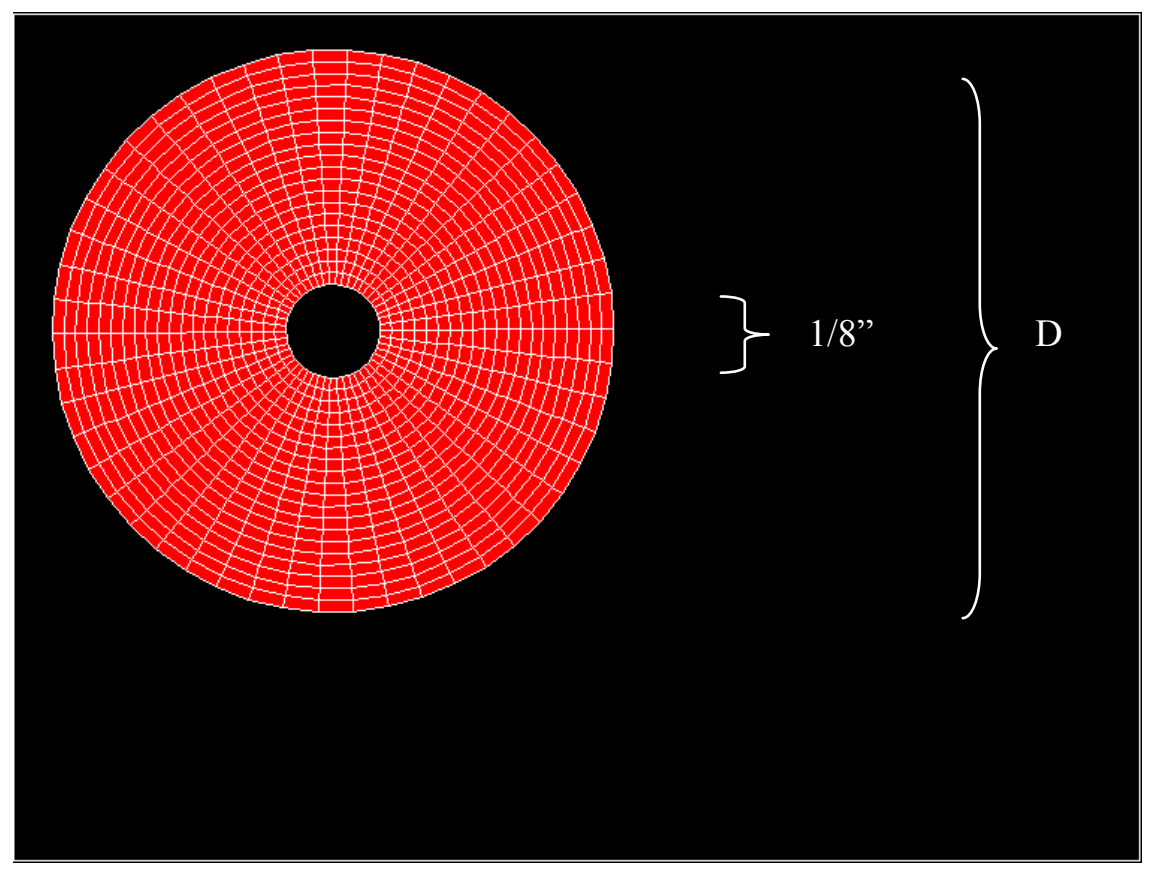

Fig. A.7 Diagram of the first slice of the separation chamber. 


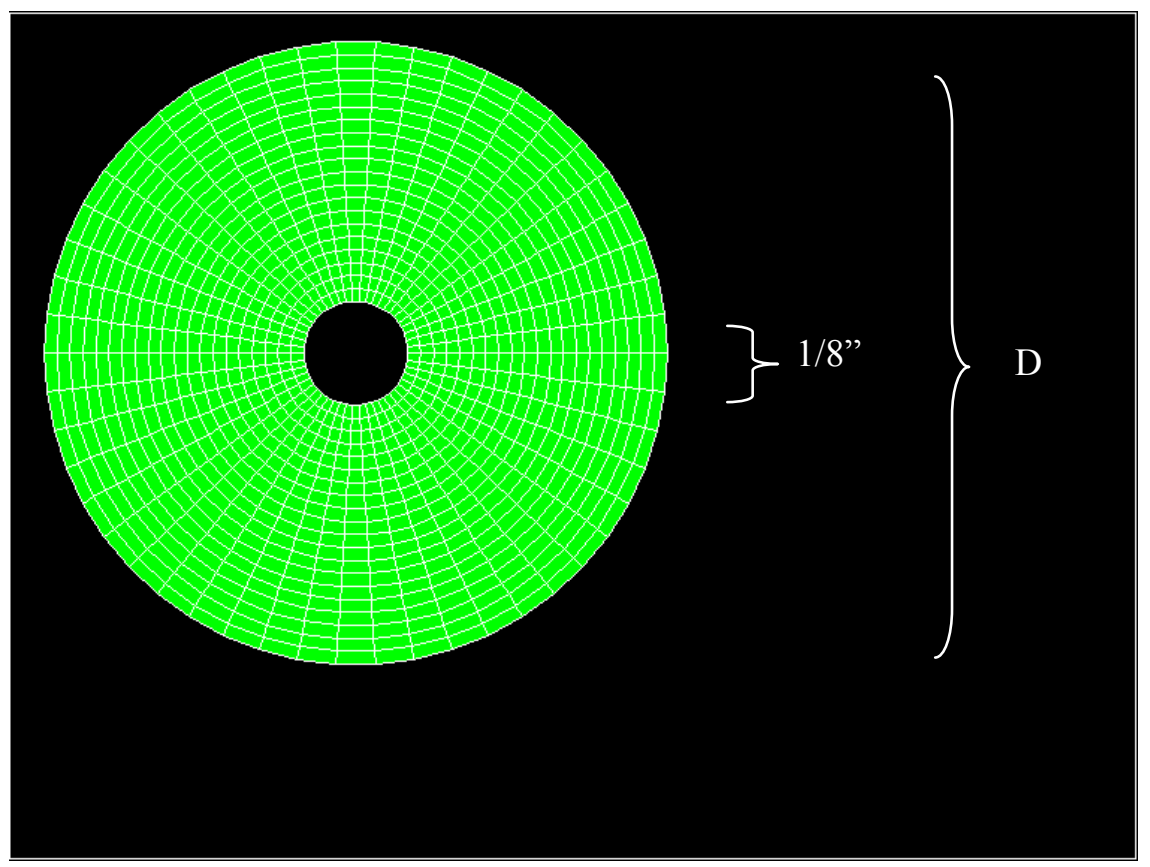

Fig. A.8 Diagram of the second section of the separation chamber.

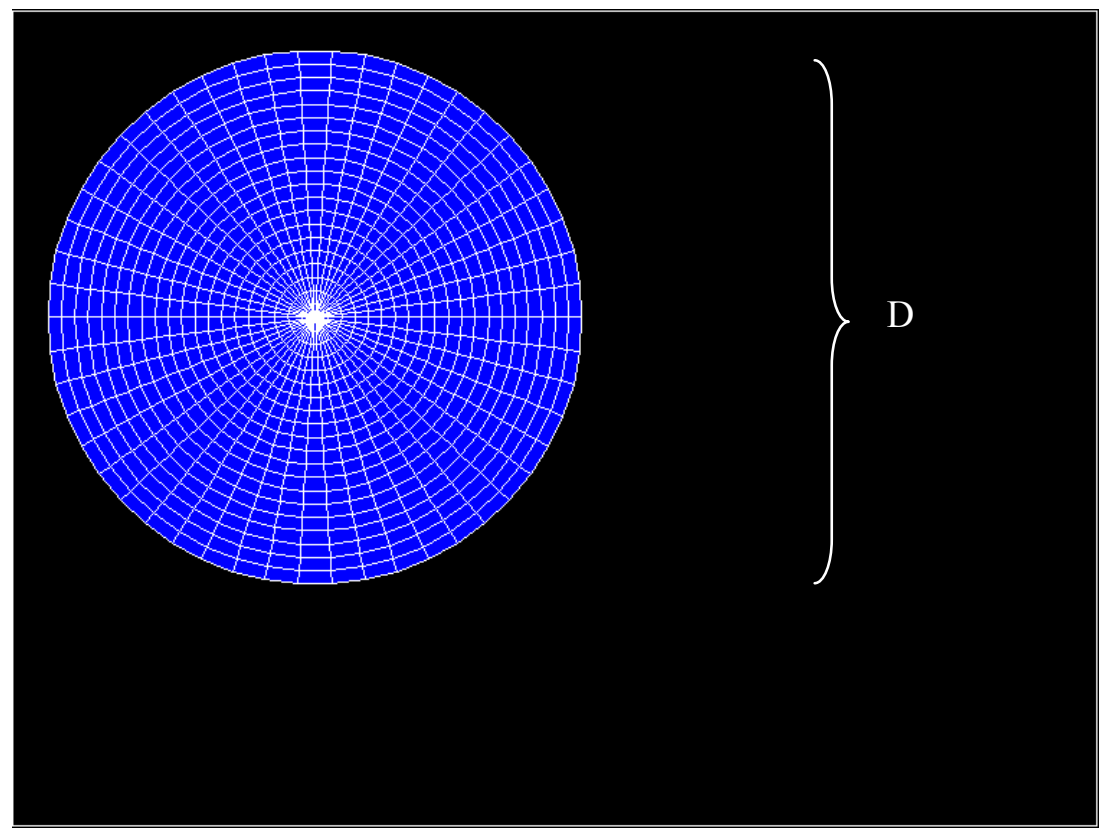

Fig. A.9 Diagram of the third portion of the separation chamber. 
The inlet and outlet boundaries of the separator were defined by selecting the faces of the cells where these boundaries belong. The inlet nozzle was constructed with an entrance region that was twice the diameter of the orifice opening from which the gas was ejected. Fig. A.10 shows a diagram of the entire separator and the locations of the inlet and outlet boundaries. The nozzle region was meshed along with the rest of the vortex generator by using the automesh tool. Around this area, the mesh size is considerably smaller than the inner portions of the vortex generator and sufficient accuracy is achieved. The cell faces that define the inlet are at the end of the larger part of the nozzle. The outlet of the separator is defined by the bottom of the blue region of the separation chamber. There are 150 cell faces that were selected as the outlet boundary.

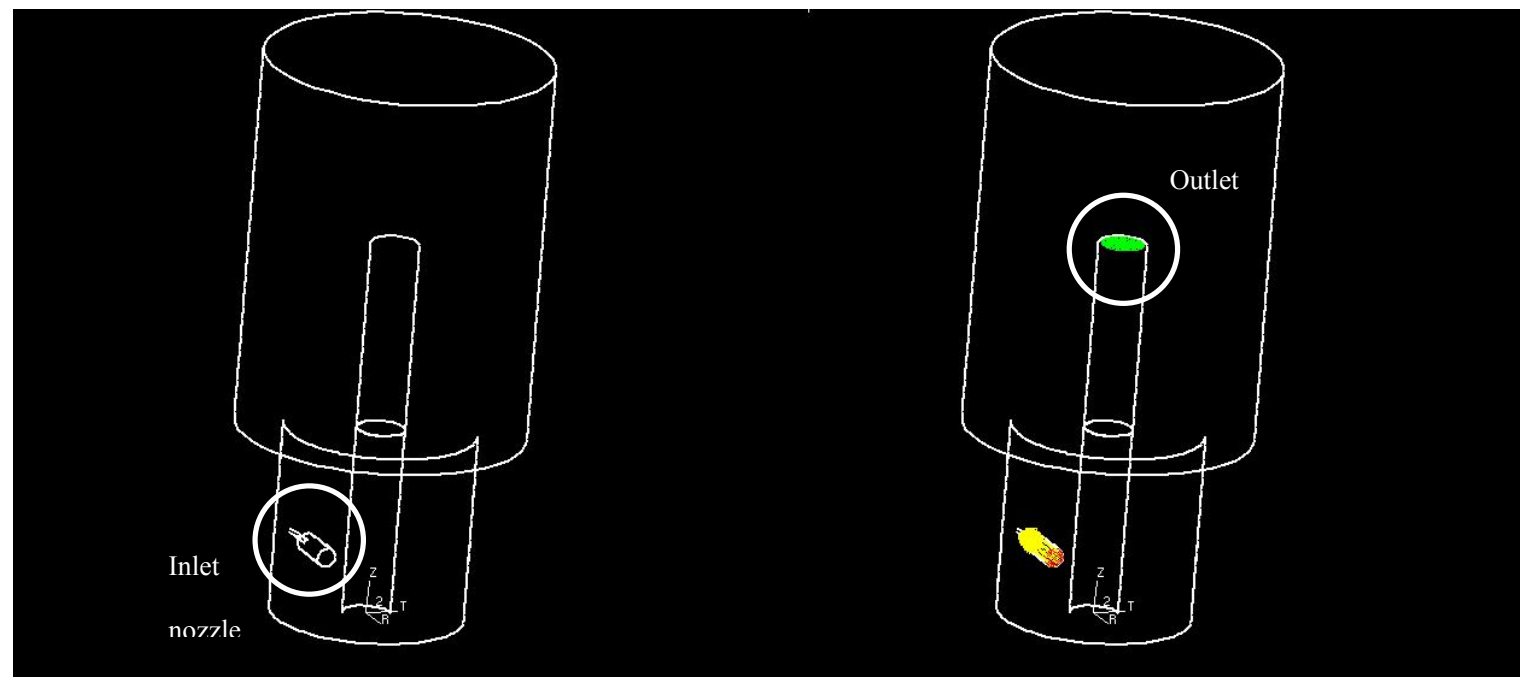

Fig. A.10 Overall geometry of the separator with the boundary regions identified

In total, there were eleven different separator geometries created in pro-STAR which mirrored the eleven tested experimentally. Each geometry had similar construction to that pictured in Fig. A.10. The next section describes the testing procedure used in the experiments and in the CFD computations. 


\section{CFD Procedure}

The separator was created as described in the Theory section. The sizes of the components of each separator created are listed in Table A.1. For each sized separator, a similar procedure to the experiment was performed. The inlet gas flow rate was ranged from 1-10 SLPM, and the free surface shape was observed at different timesteps at each flow rate. The inlet conditions for proSTAR need a gas velocity instead of volumetric flow rate, so this was furnished. The gas inlet cross sectional area was not changed when a different orifice was used. Instead, the entrance region close to the separator was adjusted to the appropriate orifice size (see Fig. A.10). The gas outlet was always set to atmospheric conditions.

Table A.1 Sizes of the components of each separator

\begin{tabular}{|c|c|c|c|c|}
\hline $\begin{array}{l}\text { Orifice Diameter } \\
\qquad \text { (m) }\end{array}$ & $\begin{array}{l}\text { Separator } \\
\text { Number }\end{array}$ & $\begin{array}{c}\text { Vortex Generator } \\
\text { Diameter (m) }\end{array}$ & $\begin{array}{c}\text { Separation } \\
\text { Chamber Diameter } \\
\text { (m) }\end{array}$ & Filename \\
\hline 0.00030 & 1 & 0.0111125 & 0.0254 & \\
\hline 0.00030 & 2 & 0.0111125 & 0.0222225 & \\
\hline 0.00030 & 3 & 0.0111125 & 0.01905 & 716vg34sc_refine.ccm \\
\hline 0.00030 & 4 & 0.0127 & 0.022225 & \\
\hline 0.00030 & 5 & 0.0127 & 0.01905 & 12vg34sc_refine.ccm \\
\hline 0.00030 & 6 & 0.009525 & 0.01905 & 38vg34sc_refine.ccm \\
\hline 0.00030 & 7 & 0.009525 & 0.015875 & \\
\hline
\end{tabular}




\begin{tabular}{|l|c|c|c|c|}
\hline 0.00025 & 8 & 0.0111125 & 0.0254 & \\
\hline 0.00025 & 9 & 0.0111125 & 0.022225 & \\
& & & & \\
\hline 0.00025 & 10 & 0.0111125 & 0.01905 & \\
& & & & \\
\hline 0.00025 & 11 & 0.009525 & 0.01905 & \\
& & & & \\
\hline
\end{tabular}

The properties of air and water used in the simulations are listed in Table A.2. These properties were initial conditions at time equal to zero and at the gas inlet. The properties of the fluids inside the separator were calculated based on the equations in the Theory section.

Table A.2 Properties of air and water used in pro-STAR

\begin{tabular}{|c|c|c|}
\hline Property & Air & Water \\
\hline Density & $1.205 \mathrm{~kg} / \mathrm{m}^{3}$ & $998.2 \mathrm{~kg} / \mathrm{m}^{3}$ \\
\hline Molecular Viscosity & & $0.001002 \mathrm{~kg} / \mathrm{m}^{*} \mathrm{~s}$ \\
\hline Specific Heat & $1.81 \mathrm{e}-5 \mathrm{~kg} / \mathrm{m}^{*} \mathrm{~s}$ & $4183 \mathrm{~J} / \mathrm{kg} * \mathrm{~K}$ \\
\hline Thermal Conductivity & $1006 \mathrm{~J} / \mathrm{kg}^{*} \mathrm{~K}$ & $0.603 \mathrm{~W} / \mathrm{m}^{*} \mathrm{~K}$ \\
\hline Inlet Temperature & & $273 \mathrm{~K}$ \\
\hline
\end{tabular}


Pro-STAR was used as the post processor to view the results. Due to the complexity of the problem being solved, convergence became an issue. The default number of outer iterations used by pro-STAR is 20 , but this was increased to 40 for every case to improve convergence. 


\section{APPENDIX B}

\section{STATISTICAL ANALYSIS OF FLOW RATE ERROR}

The experimental errors shown in the error bars presented in the figures of the Results section were the result of the inherent measurement uncertainties of the flow meter used and the coarseness of the flow rate increment used in the experiments. The flow rates measured in the flight testing were measured with a frequency of $10 \mathrm{~Hz}$, so these measurements are used for the statistical analysis and applied to the ground experiments. There were multiple flow rates measured during the flight experiments, so only one subset of the measurements is used for this statistical analysis. This subset was the data taken during the second flight day in parabola 11. This parabola had an averaged inlet gas flow rate of 7.703 SLPM with a stable gas core in the separator. In all, 498 points are used for the analysis and they are shown in the Statistical Data section below. The mean and standard deviation for these measurements are presented in Table B.1. The measurement error from the flow meter is the standard deviation $\sigma_{m}$.

Table B.1 Mean and standard deviation of measurements

\begin{tabular}{|l|l|}
\hline Mean, $\bar{x}_{m}$ & 7.703 SLPM \\
\hline Standard Deviation, $\sigma_{m}$ & 0.006732 SLPM \\
\hline
\end{tabular}

$$
\sigma_{t}=\sqrt{\sigma_{m}^{2}+\sigma_{i}^{2}}
$$


The error from the coarseness of the flow rate increment being 1 SLPM, $\sigma_{i}$, is half of this increment, 0.5 SLPM. Combining the errors in quadrature by Eq.(B.1), the total error, $\sigma_{t}$ is 0.5000. Since $\sigma_{i}$ is orders of magnitude higher than $\sigma_{m}, \sigma_{m}$ was not calculated for each tested flow rate, and $\sigma_{i}$ was applied to all of the experimental measurements.

\section{Statistical Data}
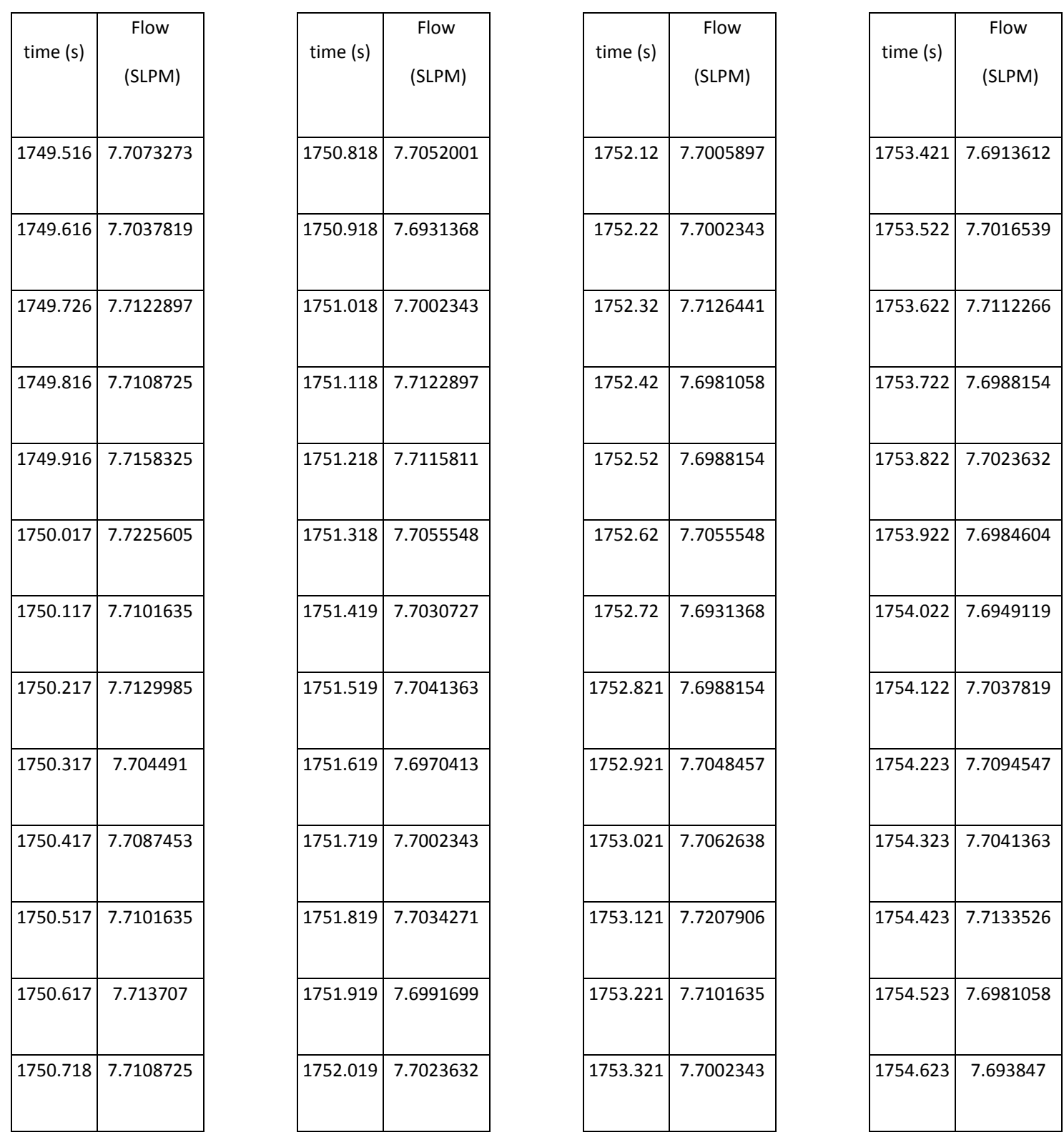

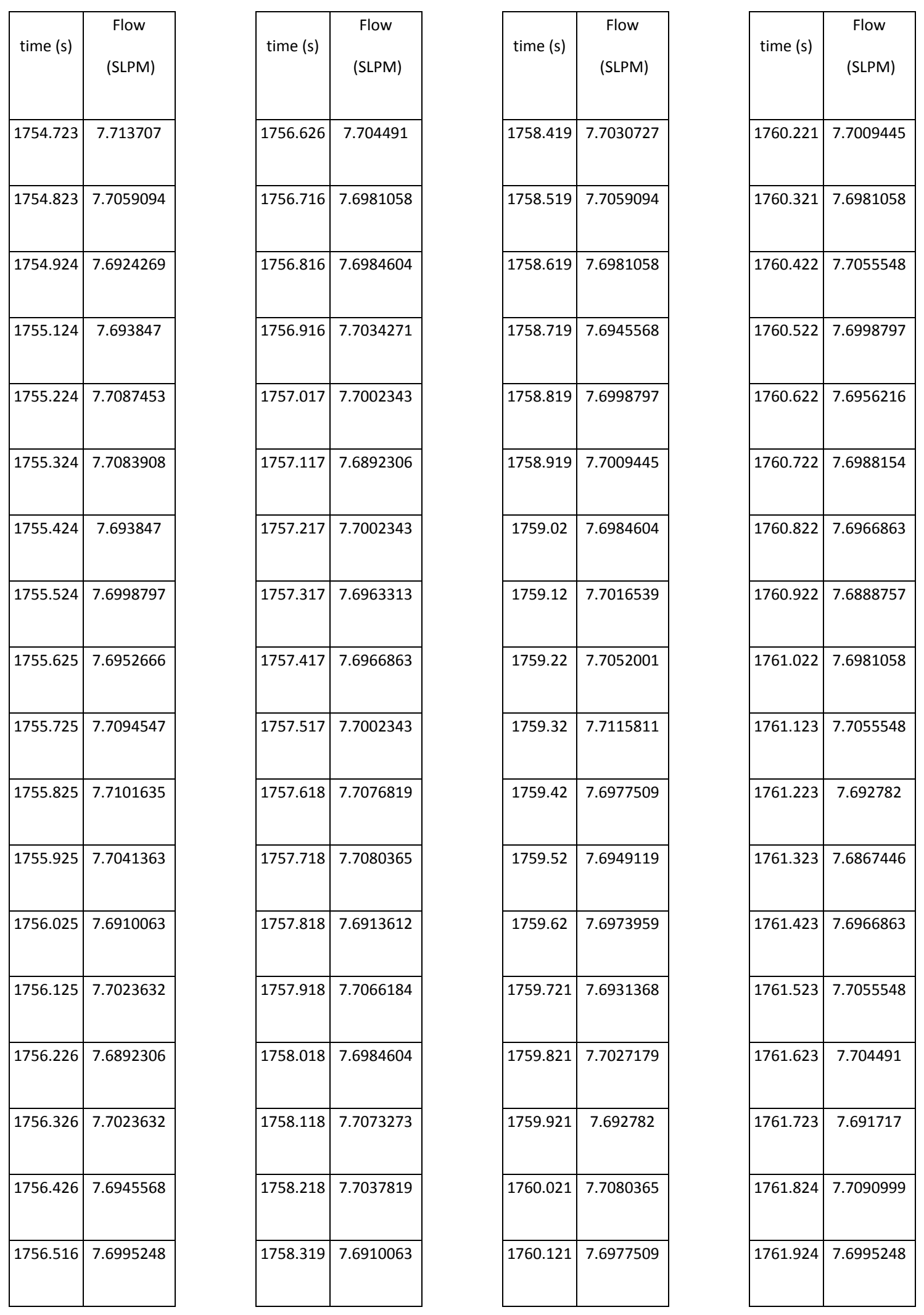

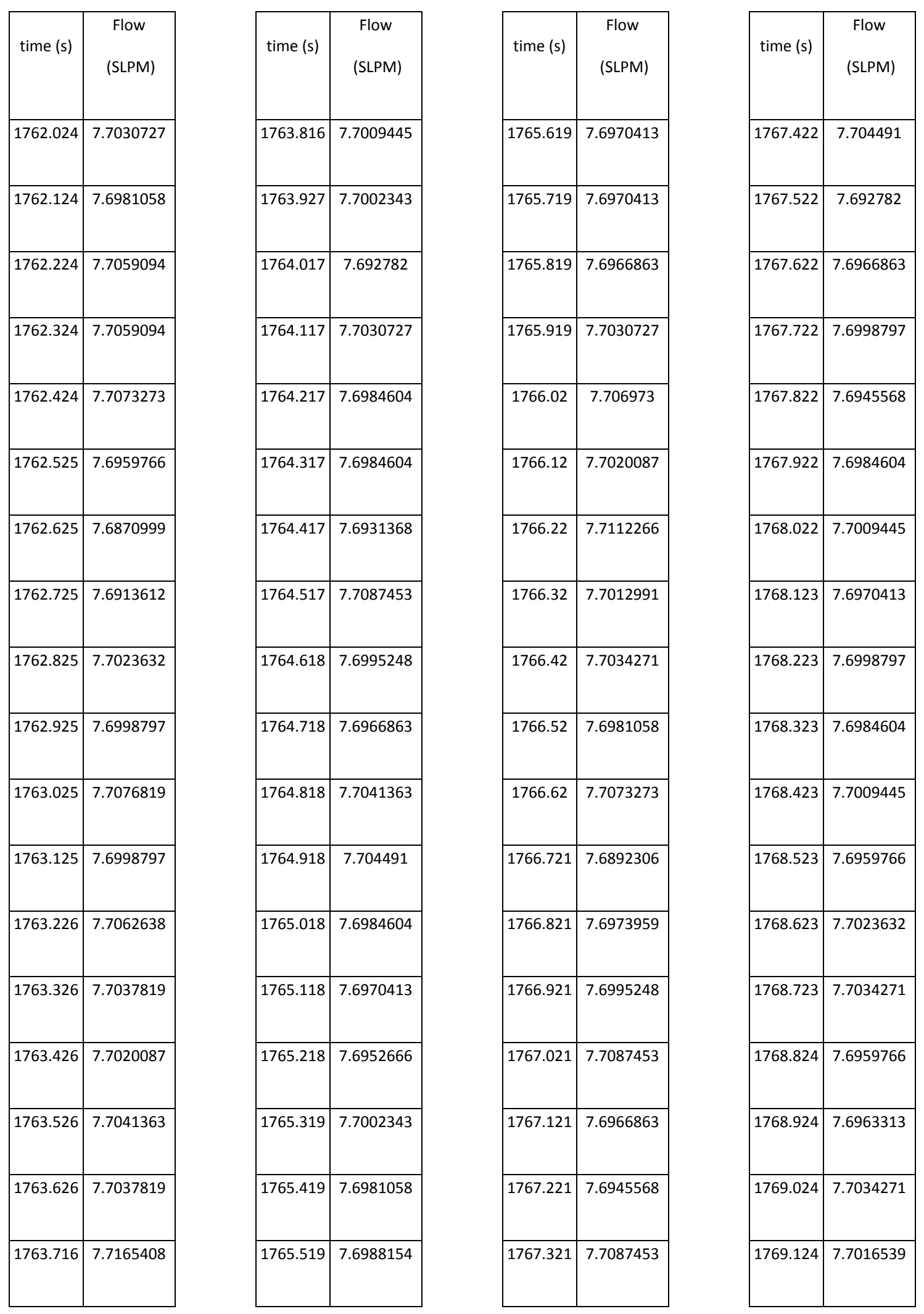

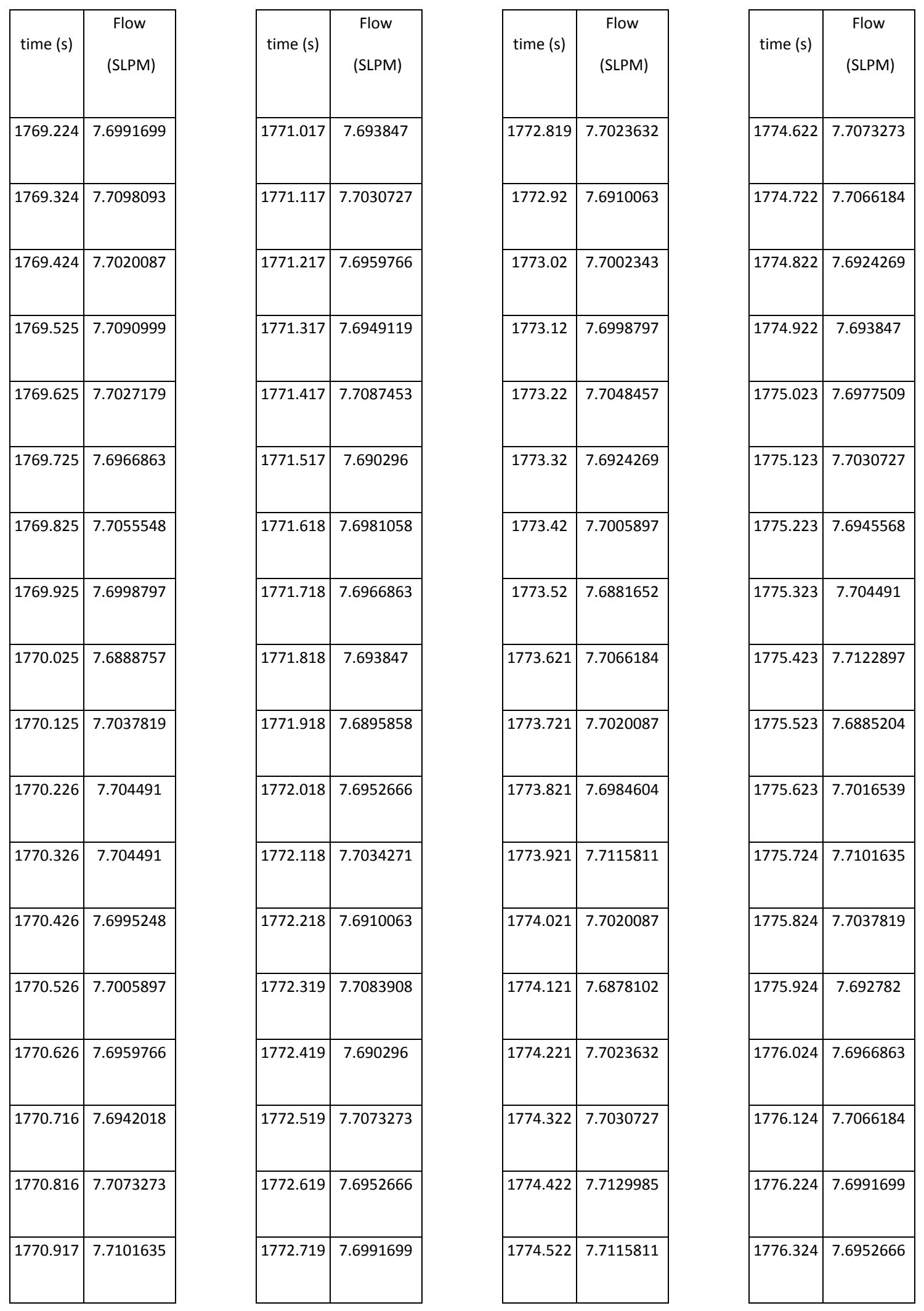

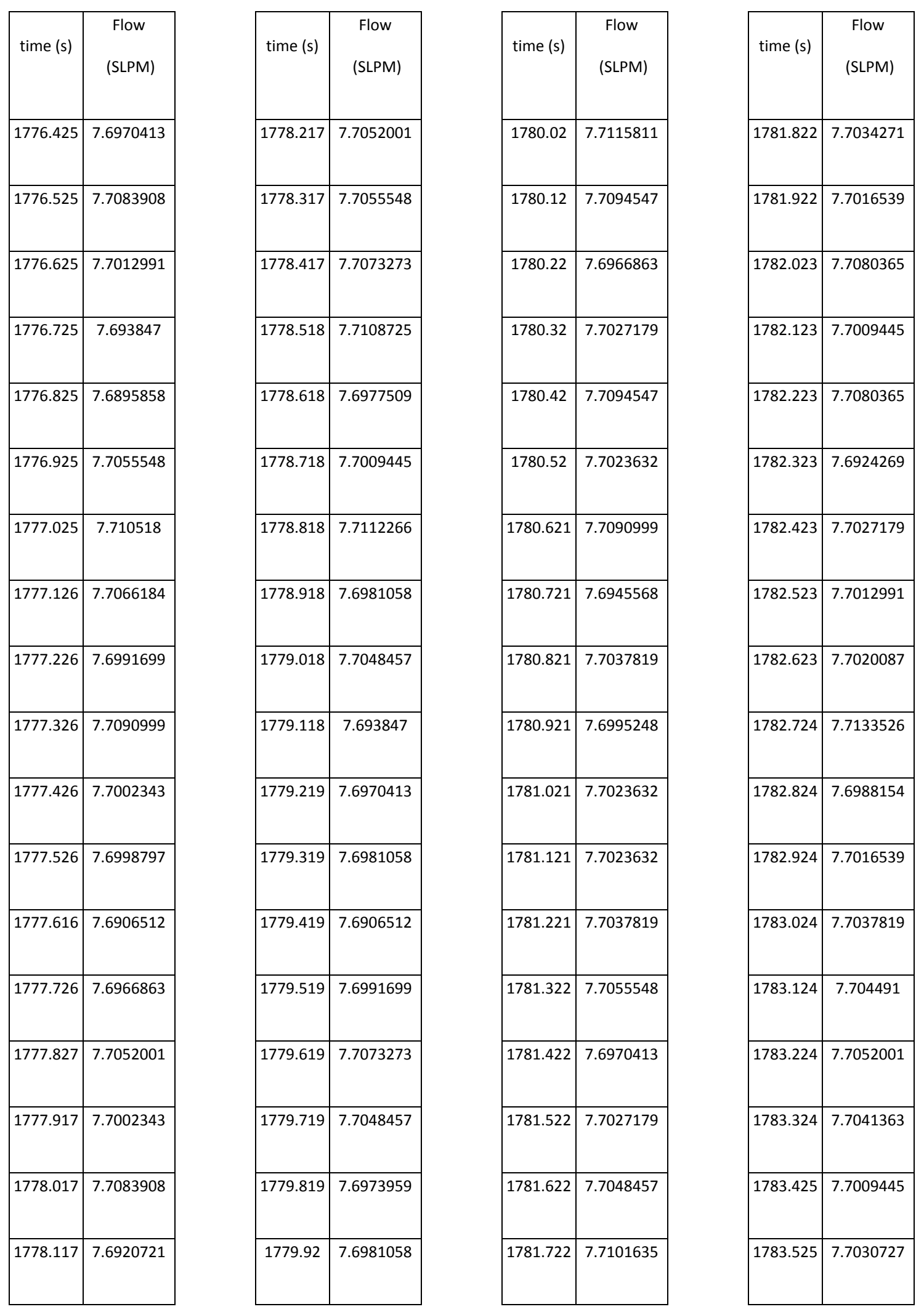

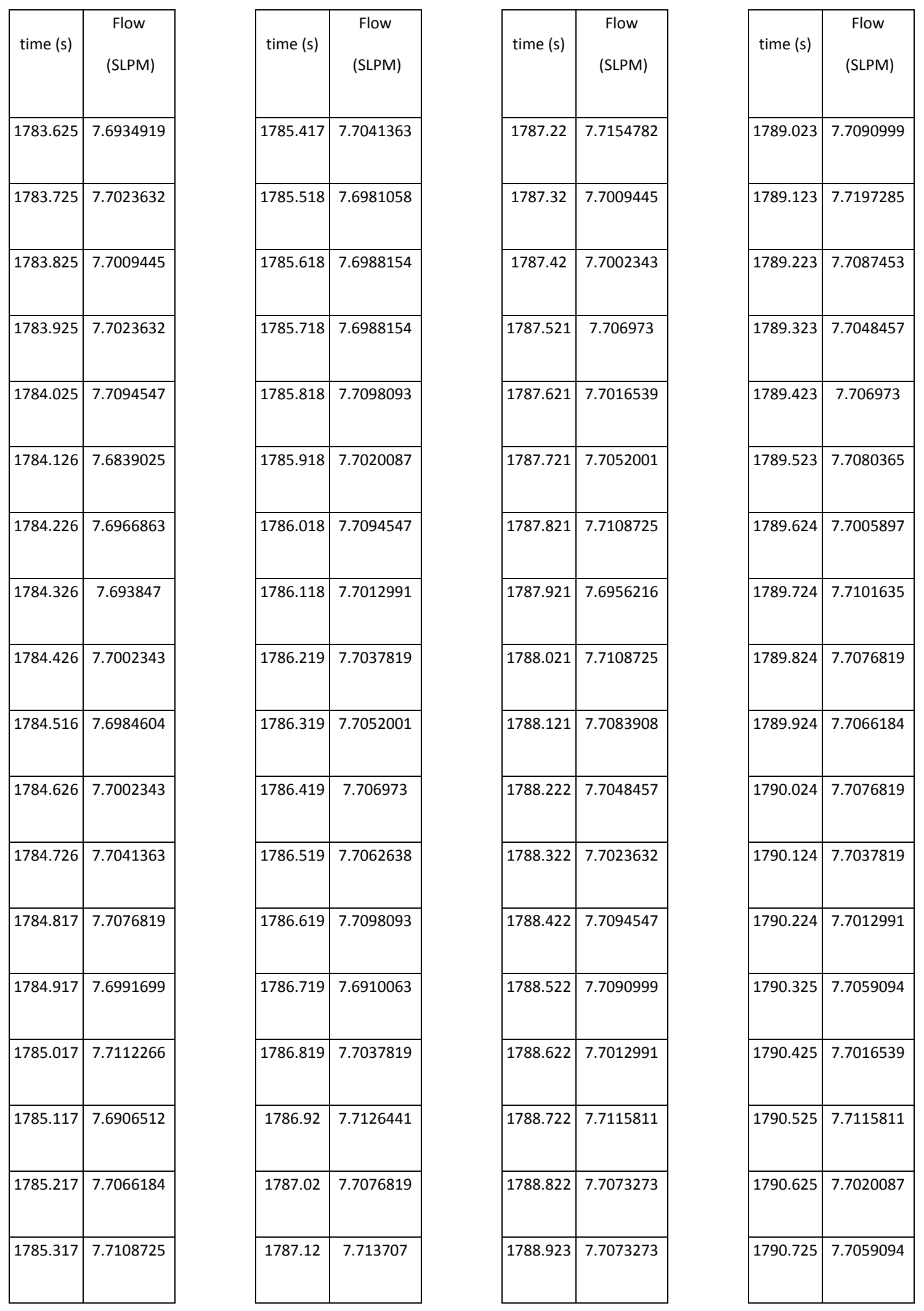

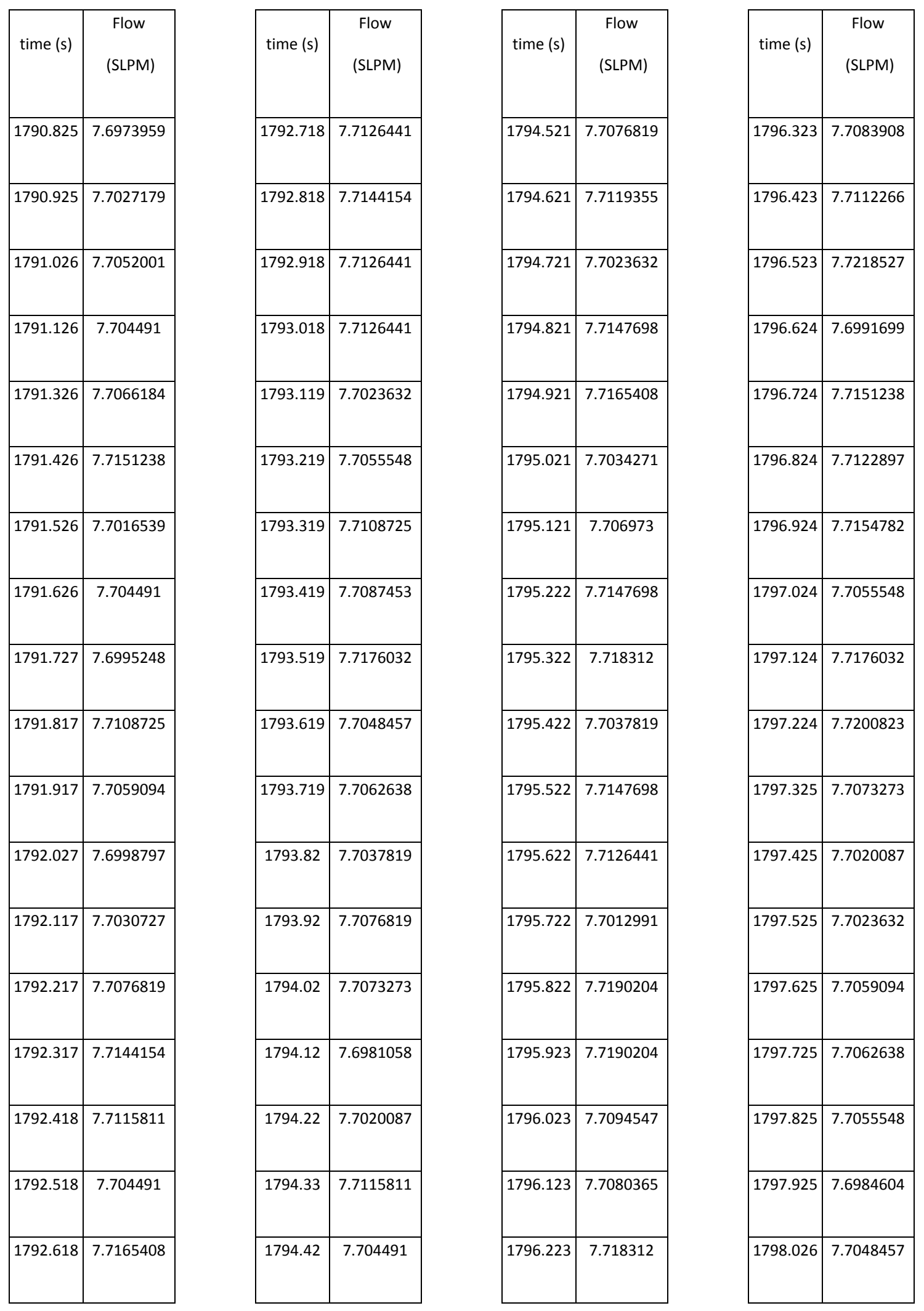


\begin{tabular}{|c|c|}
\hline time $(\mathrm{s})$ & $\begin{array}{l}\text { Flow } \\
\text { (SLPM) }\end{array}$ \\
\hline 1798.126 & 7.7023632 \\
\hline 1798.226 & 7.7144154 \\
\hline 1798.326 & 7.7020087 \\
\hline 1798.426 & 7.7059094 \\
\hline 1798.526 & 7.7133526 \\
\hline 1798.626 & 7.7062638 \\
\hline 1798.717 & 7.7154782 \\
\hline 1798.827 & 7.7098093 \\
\hline 1798.927 & 7.7101635 \\
\hline 1799.027 & 7.7052001 \\
\hline 1799.117 & 7.7041363 \\
\hline 1799.217 & 7.7059094 \\
\hline
\end{tabular}




\section{VITA}

Name:

Address:

Email Address:

Education:
Casey Klein

526 South Church Street Charlotte, NC 28202

caseyklein@gmail.com

B.S., Nuclear Engineering, Texas A\&M University, 2006 M.S., Nuclear Engineering, Texas A\&M University, 2009 